\title{
A REPRESENTATION THEOREM FOR LOCALLY COMPACT QUANTUM GROUPS
}

\author{
MARIUS JUNGE \\ Department of Mathematics \\ University of Illinois \\ Urbana, IL 61801, USA \\ junge@math.uiuc.edu \\ MATTHIAS NEUFANG \\ School of Mathematics and Statistics \\ Carleton University, Ottawa \\ Ontario, Canada K1S 5B6 \\ mneufang@math.carleton.ca \\ ZHONG-JIN RUAN \\ Department of Mathematics \\ University of Illinois \\ Urbana, IL 61801, USA \\ ruan@math.uiuc.edu
}

Received 28 January 2008

\begin{abstract}
Recently, Neufang, Ruan and Spronk proved a completely isometric representation theorem for the measure algebra $M(G)$ and for the completely bounded (Herz-Schur) multiplier algebra $M_{\mathrm{cb}} A(G)$ on $\mathcal{B}\left(L_{2}(G)\right)$, where $G$ is a locally compact group. We unify and generalize both results by extending the representation to arbitrary locally compact quantum groups $\mathbb{G}=(M, \Gamma, \varphi, \psi)$. More precisely, we introduce the algebra $M_{\mathrm{cb}}^{r}\left(L_{1}(\mathbb{G})\right)$ of completely bounded right multipliers on $L_{1}(\mathbb{G})$ and we show that $M_{\mathrm{cb}}^{r}\left(L_{1}(\mathbb{G})\right)$ can be identified with the algebra of normal completely bounded $\hat{M}$-bimodule maps on $\mathcal{B}\left(L_{2}(\mathbb{G})\right)$ which leave the subalgebra $M$ invariant. From this representation theorem, we deduce that every completely bounded right centralizer of $L_{1}(\mathbb{G})$ is in fact implemented by an element of $M_{\mathrm{cb}}^{r}\left(L_{1}(\mathbb{G})\right)$. We also show that our representation framework allows us to express quantum group "Pontryagin" duality purely as a commutation relation.
\end{abstract}

Keywords: Locally compact quantum group; completely bounded multiplier algebra; completely bounded module maps; completely isometric representation.

Mathematics Subject Classification 2000: 22D15, 22D20, 22D25, 22D35, 43A10, 43A22, 46L07, 46L10, 46L89, 47L10, 47L25 


\section{Introduction}

Let $G$ be a locally compact group. For each measure $\mu \in M(G)$, we may define a completely bounded map $\Theta^{r}(\mu)$ on $\mathcal{B}\left(L_{2}(G)\right)$ by letting

$$
\Theta^{r}(\mu)(x)=\int_{G} \rho(s) x \rho(s)^{*} d \mu(s),
$$

where $\rho$ denotes the right regular representation of $G$. It was shown by Neufang [18] (cf. also $[19,20,22])$ that $\Theta^{r}$ determines a completely isometric algebra isomorphism

$$
\Theta^{r}: M(G) \cong \mathcal{C B}_{L(G)}^{\sigma, L_{\infty}(G)}\left(\mathcal{B}\left(L_{2}(G)\right)\right)
$$

from the measure algebra $M(G)$ onto the algebra $\mathcal{C B}_{L(G)}^{\sigma, L_{\infty}(G)}\left(\mathcal{B}\left(L_{2}(G)\right)\right)$ of all normal completely bounded $L(G)$-bimodule maps on $\mathcal{B}\left(L_{2}(G)\right)$ which map $L_{\infty}(G)$ into $L_{\infty}(G)$. A corresponding result for the completely bounded (Herz-Schur) multiplier algebra $M_{\mathrm{cb}} A(G)$ has been proved by Neufang, Ruan and Spronk [22], i.e. there exists a completely isometric algebra isomorphism

$$
\hat{\Theta}: M_{\mathrm{cb}} A(G) \cong \mathcal{C B}_{L_{\infty}(G)}^{\sigma, L(G)}\left(\mathcal{B}\left(L_{2}(G)\right)\right)
$$

from $M_{\mathrm{cb}} A(G)$ onto the algebra $\mathcal{C B}_{L_{\infty}(G)}^{\sigma, L(G)}\left(\mathcal{B}\left(L_{2}(G)\right)\right)$ of all normal completely bounded $L_{\infty}(G)$-bimodule maps on $\mathcal{B}\left(L_{2}(G)\right)$ which map $L(G)$ into $L(G)$. Since we may regard the Fourier algebra $A(G)$ (respectively, its completely bounded multiplier algebra $M_{\mathrm{cb}} A(G)$ ) as the natural dual object of the convolution algebra $L_{1}(G)$ (respectively, the measure algebra $M(G)$ ), (1.2) and (1.3) show that both $M(G)$ and $M_{\mathrm{cb}} A(G)$ can be nicely represented on the same space $B\left(L_{2}(G)\right)$ in a form that perfectly displays their duality. The main purpose of this paper is to generalize the above representation theorems to arbitrary locally compact quantum groups. We remark that the question whether such a representation result holds in the setting of Kac algebras, has been raised in [18]. Further developments of this representation framework for related algebras over locally compact groups are presented, for instance, in $[25,13]$.

Locally compact quantum groups (a topological version of quantum groups) have attracted the attention of mathematicians since Drinfel'd's ICM talk [5] and Woronowicz's discovery of the twisted $S U_{q}(2)$ group [32]. There are several different definitions of locally compact quantum groups given by Baaj and Skandalis [1], Woronowicz [33], and Kustermans and Vaes in the $C^{*}$-algebra setting [15] and in the von Neumann algebra setting [16]. For compact quantum groups and discrete quantum groups, these definitions coincide with those given by Woronowicz [34], Effros and Ruan [7], and Van Daele [29]. These definitions are also all equivalent when restricted to Kac algebras.

We will use Kustermans and Vaes' notion of locally compact quantum groups $\mathbb{G}=(M, \Gamma, \varphi, \psi)$, where $(M, \Gamma)$ is a Hopf-von Neumann algebra together with a left invariant Haar weight $\varphi$ and a right invariant Haar weight $\psi$. Kac algebras can be characterized precisely as the locally compact quantum groups that admit a unitary 
antipode $\kappa$ on $M$, and whose modular element (i.e. the Radon-Nikodym derivative of the left Haar weight with respect to the right Haar weight) is affiliated with the center of $M$ (cf. [15, 27]). We will recall some basic facts about locally compact quantum groups from [16, 27 and 30] in Sec. 2. For details on Kac algebras, we refer the reader to Enock and Schwartz's book [9].

Let $\mathbb{G}=(M, \Gamma, \varphi, \psi)$ be a locally compact quantum group. There exists an operator space matrix norm on the predual $M_{*}$ of $M$, which we will denote by $L_{1}(\mathbb{G})$ (see the discussion at the end of Sec. 2). We will also write $L_{\infty}(\mathbb{G})$ for $M$. The co-multiplication $\Gamma$ induces an associative completely contractive multiplication

$$
\star=\Gamma_{*}: f_{1} \otimes f_{2} \in L_{1}(\mathbb{G}) \hat{\otimes} L_{1}(\mathbb{G}) \rightarrow f_{1} \star f_{2}=\Gamma_{*}\left(f_{1} \otimes f_{2}\right) \in L_{1}(\mathbb{G}) .
$$

With this multiplication, $L_{1}(\mathbb{G})$ is a completely contractive Banach algebra. If $G$ is a locally compact group, we get a commutative locally compact quantum group $\mathbb{G}_{a}=\left(L_{\infty}(G), \Gamma_{a}, \varphi_{a}, \psi_{a}\right)$. The co-multiplication $\Gamma_{a}$ is given by $\Gamma_{a}(f)(s, t)=f(s t)$ and the left (respectively, right) Haar weight $\varphi_{a}$ (respectively, $\psi_{a}$ ) is given by the integral with respect to a left (respectively, right) Haar measure on $G$. In this case, $L_{1}\left(\mathbb{G}_{a}\right)=L_{1}(G)$ is just the usual convolution algebra. The dual quantum group $\hat{\mathbb{G}}_{a}=\left(L(G), \hat{\Gamma}_{a}, \hat{\varphi}_{a}, \hat{\psi}_{a}\right)$ is given by the co-commutative Hopf-von Neumann algebra $\left(L(G), \hat{\Gamma}_{a}\right)$ with the plancherel weight $\hat{\varphi}_{a}=\hat{\psi}_{a}$. Then $L_{1}\left(\hat{\mathbb{G}}_{a}\right)=A(G)$ is the Fourier algebra.

Given a locally compact quantum group $\mathbb{G}$, there are two fundamental unitary operators $W$ and $V$ on the Hilbert space tensor product $L_{2}(\mathbb{G}) \otimes L_{2}(\mathbb{G})$ such that the co-multiplication $\Gamma$ can be expressed as

$$
\Gamma(x)=W^{*}(1 \otimes x) W=V(x \otimes 1) V^{*}
$$

for all $x \in M$. In particular, if $G$ is a locally compact group, we may define

$$
W_{a} \xi(s, t)=\xi\left(s, s^{-1} t\right) \quad \text { and } \quad V_{a} \xi(s, t)=\xi(s t, t)
$$

for all $\xi \in L_{2}(G \times G)=L_{2}(G) \otimes L_{2}(G)$. Both fundamental unitary operators $W$ and $V$ are considered in the literature, depending on the favor of the authors. We note that $W$ in this paper matches the notation used in $[1,16]$. However, our $W$ corresponds to $W^{*}$ in Enock and Schwartz's book [9] and many papers on Kac algebras. We hope that this will not cause any confusion to the reader.

Using these two fundamental unitary operators, we may define two injective complete contractions

$$
\Theta_{0}^{l}: f \in L_{1}(\mathbb{G}) \rightarrow \Theta_{0}^{l}(f)=\left\langle f \otimes \iota, W^{*}(1 \otimes \cdot) W\right\rangle \in \mathcal{C B}^{\sigma}\left(\mathcal{B}\left(L_{2}(\mathbb{G})\right)\right)
$$

and

$$
\Theta_{0}^{r}: f \in L_{1}(\mathbb{G}) \rightarrow \Theta_{0}^{r}(f)=\left\langle\iota \otimes f, V(\cdot \otimes 1) V^{*}\right\rangle \in \mathcal{C B}^{\sigma}\left(\mathcal{B}\left(L_{2}(\mathbb{G})\right)\right)
$$

from $L_{1}(\mathbb{G})$ into $\mathcal{C B}^{\sigma}\left(\mathcal{B}\left(L_{2}(\mathbb{G})\right)\right)$. We prove in Proposition 3.1 that $\Theta_{0}^{l}$ is an antihomomorphism, and $\Theta_{0}^{r}$ is a homomorphism from $L_{1}(\mathbb{G})$ into $\mathcal{C B}^{\sigma}\left(\mathcal{B}\left(L_{2}(\mathbb{G})\right)\right)$. 
Therefore, it is better to study the representation theorem associated with $\Theta_{0}^{r}$. We note that there is a connection between $W$ and $V$ given by the relation

$$
V=\sigma(1 \otimes U) W\left(1 \otimes U^{*}\right) \sigma
$$

where $U$ is a special unitary operator on $L_{2}(\mathbb{G})$ (see the definition given in Sec. 2) and $\sigma$ is the flip operator on $L_{2}(\mathbb{G}) \otimes L_{2}(\mathbb{G})$. Therefore, if we wish to use the left fundamental unitary operator $W$, we may consider the injective completely contractive homomorphism

$$
\Xi_{0}^{l}: f \in L_{1}(\mathbb{G}) \rightarrow \Xi_{0}^{l}(f)=\left\langle f \otimes \iota, W(1 \otimes \cdot) W^{*}\right\rangle=U^{*} \Theta_{0}^{r}(f)\left(U \cdot U^{*}\right) U
$$

from $L_{1}(\mathbb{G})$ into $\mathcal{C B}^{\sigma}\left(\mathcal{B}\left(L_{2}(\mathbb{G})\right)\right)$. The advantage of considering $\Theta_{0}^{r}$ (instead of $\left.\Xi_{0}^{l}\right)$ is that $V(\cdot \otimes 1) V^{*}=\Gamma(\cdot)$ is just the co-multiplication on $M$. In this case, we get

$$
\left\langle\Theta_{0}^{r}(f)(x), f^{\prime}\right\rangle=\left\langle f^{\prime} \otimes f, V(x \otimes 1) V^{*}\right\rangle=\left\langle f^{\prime} \star f, x\right\rangle
$$

for $x \in M$ and $f, f^{\prime} \in L_{1}(\mathbb{G})$. However, we have to consider a "twisted" comultiplication $W(1 \otimes \cdot) W^{*}$ for $\Xi_{0}^{l}$. Therefore, we will mainly work with $\Theta_{0}^{r}$ and the right fundamental unitary operator $V$. This matches our notation used in (1.1) where, for a locally compact group $G$, we considered the right representation $\Theta^{r}: \mu \in M(G) \rightarrow \Theta^{r}(\mu) \in \mathcal{C B}^{\sigma}\left(\mathcal{B}\left(L_{2}(G)\right)\right)$ given by

$$
\Theta^{r}(\mu)(x)=\int_{G} \rho(s) x \rho(s)^{*} d \mu(s)=\left\langle\iota \otimes \mu, V_{a}(x \otimes 1) V_{a}^{*}\right\rangle .
$$

We first show in Theorem 3.4 that if $\mathbb{G}$ is a discrete quantum group, then $\Theta_{0}^{r}$ is a completely isometric algebra isomorphism from $L_{1}(\mathbb{G})$ onto $\mathcal{C B}_{\hat{M}}^{\sigma, M}\left(\mathcal{B}\left(L_{2}(\mathbb{G})\right)\right)$. For general locally compact quantum groups, we shall show that $\mathcal{C B}_{\hat{M}}^{\sigma, M}\left(\mathcal{B}\left(L_{2}(\mathbb{G})\right)\right)$ corresponds precisely to the natural algebra $M_{\mathrm{cb}}^{r}\left(L_{1}(\mathbb{G})\right)$ of completely bounded right multipliers of $L_{1}(\mathbb{G})$, as introduced and studied in Sec. 4. Indeed, we shall also prove in this section that the latter algebra can be identified (up to a completely isometric anti-isomorphism) with the algebra of completely bounded right centralizers $C_{\mathrm{cb}}^{r}\left(L_{1}(\mathbb{G})\right)$ of $L_{1}(\mathbb{G})$. We note that completely bounded right (respectively, left and double) multipliers of $A(\mathbb{K})=L_{1}(\hat{\mathbb{K}})$ for Kac algebras $\mathbb{K}$ have been studied thoroughly by Kraus and Ruan in [14]. The results in Sec. 4 are the natural generalization and unification of the original results (1.2) and $(1.3)$, and some results contained in $[14,18,22]$, to locally compact quantum groups.

We present an application of our representation result to quantum group duality in Sec. 5. The fact that both $M_{\mathrm{cb}}^{r}\left(L_{1}(\mathbb{G})\right)$ and $M_{\mathrm{cb}}^{r}\left(L_{1}(\hat{\mathbb{G}})\right)$ are presented as maps on the same algebra $\mathcal{B}\left(L_{2}(\mathbb{G})\right)$ enables us to express their duality simply in terms of a commutation relation. We also show that $M_{\mathrm{cb}}^{r}\left(L_{1}(\mathbb{G})\right)$ equals its bicommutant taken in $\mathcal{C B}\left(\mathcal{B}\left(L_{2}(\mathbb{G})\right)\right)$. 


\section{Background on Locally Compact Quantum Groups}

Let $\mathbb{G}=(M, \Gamma, \varphi, \psi)$ be a locally compact quantum group. The left Haar weight $\varphi$ induces an inner product

$$
\left\langle\Lambda_{\varphi}(x) \mid \Lambda_{\varphi}(y)\right\rangle_{\varphi}=\varphi\left(y^{*} x\right)
$$

on $\mathfrak{N}_{\varphi}=\left\{x \in M: \varphi\left(x^{*} x\right)<\infty\right\}$. We let $L_{2}(M, \varphi)$ denote the Hilbert space completion of $\mathfrak{N}_{\varphi}$. Then we can obtain a conjugate linear isometric isomorphism $J$ on $L_{2}(M, \varphi)$ and we may use $J$ to isometrically anti-isomorphically identify $x \in M$ with $J x^{*} J \in M^{\prime}$, the commutant of $M$. In this situation, the von Neumann algebra $M$ is standardly represented on $L_{2}(M, \varphi)$.

There exists a left fundamental unitary operator $W$ on $L_{2}(M, \varphi) \otimes L_{2}(M, \varphi)$ associated with $\varphi$, which is defined by

$$
W^{*}\left(\Lambda_{\varphi}(x) \otimes \Lambda_{\varphi}(y)\right)=\left(\Lambda_{\varphi} \otimes \Lambda_{\varphi}\right)(\Gamma(y)(x \otimes 1))
$$

for all $x, y \in \mathfrak{N}_{\varphi}$. This operator $W$ satisfies the pentagonal relation

$$
W_{12} W_{13} W_{23}=W_{23} W_{12}
$$

and thus is a multiplicative unitary on $L_{2}(M, \varphi) \otimes L_{2}(M, \varphi)$, where we let $W_{12}=$ $W \otimes 1, W_{23}=1 \otimes W$ and $W_{13}=(\sigma \otimes 1) W_{23}(\sigma \otimes 1)$, and we let $\sigma$ be the flip map on $L_{2}(M, \varphi) \otimes L_{2}(M, \varphi)$. The co-multiplication $\Gamma$ on $M$ can be written as

$$
\Gamma(x)=W^{*}(1 \otimes x) W .
$$

The left regular representation $\lambda: L_{1}(\mathbb{G}) \rightarrow \mathcal{B}\left(L_{2}(M, \varphi)\right)$ is defined by

$$
\lambda(f)=(f \otimes \iota)(W) .
$$

It is easy to verify that $\lambda$ is an injective completely contractive homomorphism from $L_{1}(\mathbb{G})$ into $\mathcal{B}\left(L_{2}(M, \varphi)\right)$, and it is known that $\hat{M}=\left\{\lambda(f): f \in M_{*}\right\}^{\prime \prime}$ is a von Neumann algebra on $L_{2}(M, \varphi)$.

We obtain the dual quantum group $\hat{\mathbb{G}}=(\hat{M}, \hat{\Gamma}, \hat{\varphi}, \hat{\psi})$ with co-multiplication $\hat{\Gamma}$ given by

$$
\hat{\Gamma}(\hat{x})=\hat{W}^{*}(1 \otimes \hat{x}) \hat{W}
$$

where the fundamental unitary operator $\hat{W}$ is given by $\hat{W}=\sigma W^{*} \sigma$. In this case, we also get the conjugate linear isometry $\hat{J}$ on $L_{2}(M, \varphi) \cong L_{2}(\hat{M}, \hat{\varphi})$ and the completely contractive (left) regular representation

$$
\hat{\lambda}: \hat{f} \in L_{1}(\hat{\mathbb{G}}) \rightarrow(\hat{f} \otimes \iota)(\hat{W})=(\iota \otimes \hat{f})\left(W^{*}\right) \in M .
$$

It turns out that $\hat{\hat{M}}=\left\{\hat{\lambda}(\hat{f}): \hat{f} \in \hat{M}_{*}\right\}^{\prime \prime}=M$ and thus we obtain the perfect duality $\hat{\hat{G}}=\mathbb{G} !$

Similarly, the right Haar weight $\psi$ induces an inner product

$$
\left\langle\Lambda_{\psi}(x) \mid \Lambda_{\psi}(y)\right\rangle_{\psi}=\psi\left(y^{*} x\right)
$$


on $\mathfrak{N}_{\psi}=\left\{x \in M: \psi\left(x^{*} x\right)<\infty\right\}$. We let $L_{2}(M, \psi)$ denote the Hilbert space completion of $\mathfrak{N}_{\psi}$. There exists a right fundamental unitary operator $V$ on $L_{2}(M, \psi) \otimes L_{2}(M, \psi)$ associated with $\psi$, which is defined by

$$
V\left(\Lambda_{\psi}(x) \otimes \Lambda_{\psi}(y)\right)=\left(\Lambda_{\psi} \otimes \Lambda_{\psi}\right)(\Gamma(x)(1 \otimes y))
$$

for all $x, y \in \mathfrak{N}_{\psi}$. This operator $V$ also satisfies the pentagonal relation (2.1), i.e.

$$
V_{12} V_{13} V_{23}=V_{23} V_{12} \text {. }
$$

Now, the co-multiplication $\Gamma$ on $M$ can be written as

$$
\Gamma(x)=V(x \otimes 1) V^{*} .
$$

It is known from $[15,16]$ (also see $[27,30]$ ) that $\hat{J}$ determines a ${ }^{*}$-antiautomorphism $R(x)=\hat{J} x^{*} \hat{J}$ on $M$ which satisfies $\Gamma \circ R=\sigma(R \otimes R) \circ \Gamma$ and is called the unitary antipode of $\mathbb{G}$. For Kac algebras, $R$ is equal to its anitpode $\kappa$. But this is not true for general locally compact quantum groups. It is easy to verify that $\psi=\varphi \circ R$ is a right Haar weight on $M$, and we will fix this $\psi$ throughout the paper. Since $\psi\left(x^{*} x\right)=\varphi\left(R(x) R(x)^{*}\right)$ for all $x \in \mathfrak{N}_{\psi}$, the map

$$
\hat{J}\left(\Lambda_{\psi}(x)\right)=\Lambda_{\varphi}\left(R\left(x^{*}\right)\right)
$$

extends to a conjugate linear isometric isomorphism from $L_{2}(M, \psi)$ onto $L_{2}(M, \varphi)$ (cf. [27, Proposition 1.13.14]). Since we may isometrically identify $L_{2}(M, \varphi)$ and $L_{2}(M, \psi)$, we will simply use the notation $L_{2}(\mathbb{G})$ for this Hilbert space.

We note that for general locally compact quantum groups, $U=\hat{J} J$ is a unitary operator on $L_{2}(\mathbb{G})$ satisfying

$$
\hat{J} J=v^{\frac{i}{4}} J \hat{J}
$$

(cf. [27, Corollary 1.13.15]). Since

$$
(\hat{J} \otimes J) W(\hat{J} \otimes J)=W^{*},
$$

$V$ and $W$ are related by the following formula

$$
V=\sigma(\hat{J} \otimes \hat{J}) W^{*}(\hat{J} \otimes \hat{J}) \sigma=\sigma(1 \otimes U) W\left(1 \otimes U^{*}\right) \sigma .
$$

Since $W \in M \bar{\otimes} \hat{M}$, we get $V \in \hat{M}^{\prime} \bar{\otimes} M$ and

$$
(J \otimes \hat{J}) V(J \otimes \hat{J})=V^{*} .
$$

The right regular representation $\rho: L_{1}(\mathbb{G}) \rightarrow \mathcal{B}\left(L_{2}(\mathbb{G})\right)$ can be defined by

$$
\rho(f)=(\iota \otimes f)(V)=U \lambda(f) U^{*} \in \hat{M}^{\prime} .
$$

The corresponding dual quantum group can be expressed as $\hat{\mathbb{G}}^{\prime}=\left(\hat{M}^{\prime}, \hat{\Gamma}^{\prime}, \hat{\varphi}^{\prime}, \hat{\psi}^{\prime}\right)$, where the co-multiplication $\hat{\Gamma}^{\prime}$ is given by

$$
\hat{\Gamma}^{\prime}\left(\hat{x}^{\prime}\right)=\hat{V}\left(\hat{x}^{\prime} \otimes 1\right) \hat{V}^{*}=\sigma(\hat{J} \otimes \hat{J}) \hat{\Gamma}\left(\hat{J} \hat{x}^{\prime} \hat{J}\right)(\hat{J} \otimes \hat{J}) \sigma .
$$


In this case (by considering the duality of $\mathbb{G}$ and $\hat{\mathbb{G}}^{\prime}$ ), we have

$$
\hat{V}=\sigma(\hat{J} \otimes \hat{J}) \hat{W}^{*}(\hat{J} \otimes \hat{J}) \sigma \in M \bar{\otimes} \hat{M}^{\prime},
$$

and thus we obtain

$$
\hat{V}=(\hat{J} \otimes \hat{J}) W(\hat{J} \otimes \hat{J})=\sigma V^{*} \sigma .
$$

We note that Vaes considered

$$
\hat{V}=\sigma(J \otimes J) \hat{W}^{*}(J \otimes J) \sigma=(J \otimes J) W(J \otimes J) \in M^{\prime} \bar{\otimes} \hat{M}
$$

in [27], which is unitarily equivalent to our definition given in (2.9) via the unitary operator $U \otimes U$. But the latter does not satisfy $\hat{V}=\sigma V^{*} \sigma$. So we will use (2.9) throughout this paper.

Given a locally compact quantum group $\mathbb{G}$, we let $M, M^{\prime}, \hat{M}$ and $\hat{M}^{\prime}$ be the von Neumann algebras associated with $\mathbb{G}$ and let $\langle M \hat{M}\rangle$ denote the $\sigma$-weak closure of the linear span of $M \hat{M}=\{x \hat{y}: x \in M, \hat{y} \in \hat{M}\}$ in $\mathcal{B}\left(L_{2}(\mathbb{G})\right)$. There are two useful results for our representation theorem.

Proposition 2.1. We have

$$
M \cap \hat{M}=M^{\prime} \cap \hat{M}=M \cap \hat{M}^{\prime}=M^{\prime} \cap \hat{M}^{\prime}=\mathbb{C} 1 .
$$

Proposition 2.2. We have

$$
\left\langle\hat{M}^{\prime} M\right\rangle=\left\langle\hat{M} M^{\prime}\right\rangle=\langle M \hat{M}\rangle=\left\langle M^{\prime} \hat{M}^{\prime}\right\rangle=\mathcal{B}\left(L_{2}(\mathbb{G})\right) .
$$

Proposition 2.1 is well-known for Kac algebras (cf. Enock and Schwartz's book [9, Corollary 4.1.5]). It is also true for locally compact quantum groups (cf. Vaes and Van Daele [28] and Van Daele [30]). Since the $\sigma$-weak closure $\langle M \hat{M}\rangle$ is a von Neumann algebra and its commutant is equal to $M^{\prime} \cap \hat{M}^{\prime}=\mathbb{C} 1$, this shows that $\langle M \hat{M}\rangle=B\left(L_{2}(\mathbb{G})\right)$. This proof can be found in Vaes and van Daele [28, Proposition 2.5]. We would like to thank Leonid Vaineman to point out this reference to us.

Finally, we make the following remark on the operator space structure of $L_{1}(\mathbb{G})$. Assume that we are given an orthonormal basis $\left\{\xi_{i}\right\}$ for $L_{2}(\mathbb{G})$. Then the rank one operators $e_{i j}=\theta_{\xi_{i}} \theta_{\xi_{j}}^{*} \cong \xi_{i} \otimes \xi_{j}^{*}$; form a system of matrix unit for $\mathcal{B}\left(L_{2}(\mathbb{G})\right)$. With this matrix unit, we can obtain a canonical operator space matrix norm on $\mathcal{T}\left(L_{2}(\mathbb{G})\right)$ via the parallel duality

$$
\langle x, y\rangle=\sum_{i j} x_{i j} y_{i j}=\operatorname{tr}\left(x^{t} y\right)
$$

for $x \in \mathcal{T}\left(L_{2}(\mathbb{G})\right.$ ) and $y \in \mathcal{B}\left(L_{2}(\mathbb{G})\right.$ ) (for details, see [8]). Here, we let tr denote the trace on $\mathcal{B}\left(L_{2}(\mathbb{G})\right)$ and $x^{t}$ denote the transpose of $x$. In this case, the corresponding dual basis in $\mathcal{T}\left(L_{2}(\mathbb{G})\right)$ is given by

$$
\hat{e}_{i j}=\theta_{\xi_{i}}^{*} \theta_{\xi_{j}}=e_{i j}^{t} \cdot \operatorname{tr} .
$$


Throughout this paper, we assume that $\mathcal{T}\left(L_{2}(\mathbb{G})\right)$ is equipped with this canonical operator space structure and assume that $L_{1}(\mathbb{G})$ carries the operator space stucture stemming from the quotient space $\mathcal{T}\left(L_{2}(\mathbb{G})\right) / M_{\perp}$. With this in mind, we obtain the canonical operator space matrix norm on $S_{p}\left(L_{2}(\mathbb{G})\right)$, the Schatten $p$-classes on $L_{2}(\mathbb{G})$, via complex interpolation (see Pisier's work [23 or 24]).

\section{The Representation for Discrete Quantum Groups}

Let us first consider the maps $\Theta_{0}^{l}$ and $\Theta_{0}^{r}$ defined in (1.6) and (1.7).

Proposition 3.1. Let $\mathbb{G}$ be a locally compact quantum group.

(1) $\Theta_{0}^{l}$ is an injective completely contractive anti-homomorphism from $L_{1}(\mathbb{G})$ into $\mathcal{C B}_{\hat{M}^{\prime}}^{\sigma, M}\left(\mathcal{B}\left(L_{2}(\mathbb{G})\right)\right)$.

(2) $\Theta_{0}^{r}$ is an injective completely contractive homomorphism from $L_{1}(\mathbb{G})$ into $\mathcal{C B}_{\hat{M}}^{\sigma, M}\left(\mathcal{B}\left(L_{2}(\mathbb{G})\right)\right)$.

Proof. We shall first consider $\Theta_{0}^{l}$. Since $W \in M \bar{\otimes} \hat{M}$, we get $W(1 \otimes x) W^{*} \in$ $M \bar{\otimes} \mathcal{B}\left(L_{2}(\mathbb{G})\right)$ for all $x \in \mathcal{B}\left(L_{2}(\mathbb{G})\right)$, and we obtain

$$
\begin{aligned}
\Theta_{0}^{l}\left(f_{1} \star f_{2}\right)(x) & =\left\langle f_{1} \otimes f_{2} \otimes \iota,(\Gamma \otimes \iota)\left(W^{*}(1 \otimes x) W\right)\right\rangle \\
& =\left\langle f_{1} \otimes f_{2} \otimes \iota, W_{12}^{*} W_{23}^{*}(1 \otimes 1 \otimes x) W_{23} W_{12}\right\rangle \\
& =\left\langle f_{1} \otimes f_{2} \otimes \iota, W_{23}^{*} W_{13}^{*}(1 \otimes 1 \otimes x) W_{13} W_{23}\right\rangle \\
& =\left\langle f_{2} \otimes \iota, W^{*}\left(1 \otimes \Theta_{0}^{l}\left(f_{1}\right)(x)\right) W\right\rangle=\Theta_{0}^{l}\left(f_{2}\right)\left(\Theta_{0}^{l}\left(f_{1}\right)(x)\right)
\end{aligned}
$$

for all $f_{1}, f_{2} \in L_{1}(\mathbb{G})$. This shows that $\Theta_{0}^{l}\left(f_{1} \star f_{2}\right)=\Theta_{0}^{l}\left(f_{2}\right) \circ \Theta_{0}^{l}\left(f_{1}\right)$ and thus $\Theta_{0}^{l}$ is an injective completely contractive anti-homomorphism from $L_{1}(\mathbb{G})$ into $\mathcal{C B}^{\sigma}\left(\mathcal{B}\left(L_{2}(\mathbb{G})\right)\right)$. Since $W \in M \bar{\otimes} \hat{M}$, for each $f \in L_{1}(\mathbb{G})$,

$$
\Theta_{0}^{l}(f)=\left\langle f \otimes \iota, W^{*}(1 \otimes \cdot) W\right\rangle
$$

is clearly an $\hat{M}^{\prime}$-bimodule map. Moreover, if $x \in M$ we have $W^{*}(1 \otimes x) W=\Gamma(x) \in$ $M \bar{\otimes} M$. Therefore, $\Theta_{0}^{l}(f)$ maps $M$ into $M$. This shows that $\Theta_{0}^{l}$ is an injective completely contractive anti-homomorphism from $L_{1}(\mathbb{G})$ into $\mathcal{C B}_{\hat{M}^{\prime}}^{\sigma, M}\left(\mathcal{B}\left(L_{2}(\mathbb{G})\right)\right.$ ).

Similarly, since $V(x \otimes 1) V^{*} \in \mathcal{B}\left(L_{2}(\mathbb{G})\right) \bar{\otimes} M$ for all $x \in \mathcal{B}\left(L_{2}(\mathbb{G})\right)$, we obtain

$$
\begin{aligned}
\Theta_{0}^{r}\left(f_{1} \star f_{2}\right)(x) & =\left\langle\iota \otimes f_{1} \otimes f_{2},(\iota \otimes \Gamma)\left(V(x \otimes 1) V^{*}\right)\right\rangle \\
& =\left\langle\iota \otimes f_{1} \otimes f_{2}, V_{23} V_{12}(x \otimes 1 \otimes 1) V_{12}^{*} V_{23}^{*}\right\rangle \\
& =\left\langle\iota \otimes f_{1} \otimes f_{2}, V_{12} V_{13}(x \otimes 1 \otimes 1) V_{13}^{*} V_{12}^{*}\right\rangle \\
& =\left\langle\iota \otimes f_{1}, V\left(\Theta_{0}^{r}\left(f_{2}\right)(x) \otimes 1\right) V^{*}\right\rangle=\Theta_{0}^{r}\left(f_{1}\right)\left(\Theta_{0}^{r}\left(f_{2}\right)(x)\right)
\end{aligned}
$$

for all $f_{1}, f_{2} \in L_{1}(\mathbb{G})$. 
This shows that $\Theta_{0}^{r}\left(f_{1} \star f_{2}\right)=\Theta_{0}^{r}\left(f_{1}\right) \circ \Theta_{0}^{r}\left(f_{2}\right)$, and thus $\Theta_{0}^{r}$ is an injective completely contractive homomorphism from $L_{1}(\mathbb{G})$ into $\mathcal{C B}^{\sigma}\left(\mathcal{B}\left(L_{2}(\mathbb{G})\right)\right)$. Since $V \in \hat{M}^{\prime} \bar{\otimes} M$, for each $f \in L_{1}(\mathbb{G})$,

$$
\Theta_{0}^{r}(f)=\left\langle\iota \otimes f, V(\cdot \otimes 1) V^{*}\right\rangle
$$

is an $\hat{M}$-bimodule map. Moreover, if $x \in M$ we have $V(x \otimes 1) V^{*}=\Gamma(x) \in M \bar{\otimes} M$. Therefore, $\Theta_{0}^{r}(f)$ maps $M$ into $M$. This shows that $\Theta_{0}^{r}$ is an injective completely contractive homomorphism from $L_{1}(\mathbb{G})$ into $\mathcal{C B}_{\hat{M}}^{\sigma, M}\left(\mathcal{B}\left(L_{2}(\mathbb{G})\right)\right.$ ).

Proposition 3.2. Let $\mathbb{G}$ be a locally compact quantum group. If $\Phi$ is a normal completely bounded map in $\mathcal{C B}_{\hat{M}}^{\sigma, M}\left(\mathcal{B}\left(L_{2}(\mathbb{G})\right)\right)$, then there exists a unique $\hat{b}^{\prime} \in \hat{M}^{\prime}$ such that

$$
(\iota \otimes \Phi)(V)=V\left(\hat{b}^{\prime} \otimes 1\right) .
$$

In particular, if $\Phi=\Theta_{0}^{r}(f)$ for some $f \in L_{1}(\mathbb{G})$, then $\hat{b}^{\prime}=\rho(f)$.

Proof. The idea is similar to the argument given in [22, Theorem 4.2]. Since $\Phi$ is a normal completely bounded $\hat{M}$-bimodule map on $\mathcal{B}\left(L_{2}(\mathbb{G})\right)$, it is known from an unpublished result of Haagerup [10] that there exist two nets of elements $\left\{\hat{a}_{i}^{\prime}\right\}$ and $\left\{\hat{b}_{i}^{\prime}\right\}$ in $\hat{M}^{\prime}$ such that

$$
\Phi(x)=\sum_{i \in I} \hat{a}_{i}^{\prime} x \hat{b}_{i}^{\prime},
$$

converging in the weak ${ }^{*}$ topology on $\mathcal{B}\left(L_{2}(\mathbb{G})\right)$, for all $x \in \mathcal{B}\left(L_{2}(\mathbb{G})\right)$. Now we can write

$$
\hat{V}(\Phi \otimes \iota)\left(\hat{V}^{*}\right)=\sum_{i \in I} \hat{V}\left(\hat{a}_{i}^{\prime} \otimes 1\right) \hat{V}^{*}\left(\hat{b}_{i}^{\prime} \otimes 1\right) .
$$

Since $\hat{V}\left(\hat{a}_{i}^{\prime} \otimes 1\right) \hat{V}^{*}=\hat{\Gamma}^{\prime}\left(\hat{a}_{i}^{\prime}\right) \in \hat{M}^{\prime} \bar{\otimes} \hat{M}^{\prime}$, the right-hand side of (3.2) belongs to $\hat{M}^{\prime} \bar{\otimes} \hat{M}^{\prime}$. But the left-hand side of (3.2) belongs to $M \bar{\otimes} \hat{M}^{\prime}$ because both $\hat{V}$ and $(\Phi \otimes \iota)\left(\hat{V}^{*}\right)$ belong to $M \bar{\otimes} \hat{M}^{\prime}$. It follows from Proposition 2.1 that

$$
\hat{V}(\Phi \otimes \iota)\left(\hat{V}^{*}\right) \in\left(\hat{M}^{\prime} \bar{\otimes} \hat{M}^{\prime}\right) \cap\left(M \bar{\otimes} \hat{M}^{\prime}\right)=\mathbb{C} \bar{\otimes} \hat{M}^{\prime},
$$

and thus there exists $\hat{b}^{\prime} \in \hat{M}^{\prime}$ such that

$$
\hat{V}(\Phi \otimes \iota)\left(\hat{V}^{*}\right)=1 \otimes \hat{b}^{\prime} .
$$

Since $\hat{V}^{*}=\sigma V \sigma$, we conclude that

$$
(\iota \otimes \Phi)(V)=V\left(\hat{b}^{\prime} \otimes 1\right) .
$$

The uniqueness of $\hat{b}^{\prime}$ is obvious.

Now if $\Phi=\Theta_{0}^{r}(f)$ for some $f \in L_{1}(\mathbb{G})$, then we must have $\hat{b}^{\prime}=\rho(f)$ since for all $\omega \in \mathcal{T}\left(L_{2}(\mathbb{G})\right)$ and $g \in L_{1}(\mathbb{G})$,

$$
\begin{aligned}
\left\langle\omega \otimes g,\left(\iota \otimes \Theta_{0}^{r}(f)\right)(V)\right\rangle & =\langle\omega \otimes g \star f, V\rangle=\langle\omega, \rho(g \star f)\rangle \\
& =\langle\omega, \rho(g) \rho(f)\rangle=\langle\omega \otimes g, V(\rho(f) \otimes 1)\rangle .
\end{aligned}
$$


Proposition 3.3. The induced map

$$
\Pi^{r}: \Phi \in \mathcal{C B}_{\hat{M}}^{\sigma, M}\left(\mathcal{B}\left(L_{2}(\mathbb{G})\right)\right) \rightarrow \hat{b}^{\prime} \in \hat{M}^{\prime}
$$

is a unital completely contractive algebra homomorphism.

Proof. Given $\left[\Phi_{i j}\right] \in M_{n}\left(\mathcal{C B}_{\hat{M}}^{\sigma, M}\left(\mathcal{B}\left(L_{2}(\mathbb{G})\right)\right)\right)$, we let $\left[\hat{b}_{i j}^{\prime}\right]=\left[\Pi^{r}\left(\Phi_{i j}\right)\right] \in M_{n}(M)$ and obtain

$$
\left\|\left[\hat{b}_{i j}^{\prime}\right]\right\|=\left\|\left[V\left(\hat{b}_{i j}^{\prime} \otimes 1\right)\right]\right\|=\left\|\left[\left(\iota \otimes \Phi_{i j}\right)(V)\right]\right\| \leq\left\|\left[\Phi_{i j}\right]\right\|_{\mathrm{cb}} .
$$

This shows that $\Pi^{r}$ is a complete contraction from $\mathcal{C B}_{\hat{M}}^{\sigma, M}\left(\mathcal{B}\left(L_{2}(\mathbb{G})\right)\right)$ into $\hat{M}^{\prime}$. To prove that $\Pi^{r}$ is a homomorphism, we consider $\Phi_{1}$ and $\Phi_{2}$ in $\mathcal{C B}_{\hat{M}}^{\sigma, M}\left(\mathcal{B}\left(L_{2}(\mathbb{G})\right)\right)$, and we have

$$
\left(\iota \otimes \Phi_{1} \circ \Phi_{2}\right)(V)=\left(\iota \otimes \Phi_{1}\right)\left(V\left(\hat{b}_{2}^{\prime} \otimes 1\right)\right)=V\left(\hat{b}_{1}^{\prime} \hat{b}_{2}^{\prime} \otimes 1\right) .
$$

Therefore, $\Pi^{r}\left(\Phi_{1} \circ \Phi_{2}\right)=\hat{b}_{1}^{\prime} \hat{b}_{2}^{\prime}=\Pi^{r}\left(\Phi_{1}\right) \Pi^{r}\left(\Phi_{2}\right)$.

It is easy to see from Propositions 3.2 and 3.3 that the composition map $\Pi^{r} \circ \Theta_{0}^{r}$ is simply equal to the right regular representation $\rho$, i.e. we have

$$
\rho=\Pi^{r} \circ \Theta_{0}^{r}: L_{1}(\mathbb{G}) \rightarrow \hat{M}^{\prime} .
$$

Now we are ready to prove our representation theorem for discrete quantum groups.

Theorem 3.4. Let $\mathbb{G}$ be a discrete quantum group. Then the map $\Theta_{0}^{r}$ is a completely isometric algebra isomorphism from $L_{1}(\mathbb{G})$ onto $\mathcal{C B}_{\hat{M}}^{\sigma, M}\left(\mathcal{B}\left(L_{2}(\mathbb{G})\right)\right)$.

Proof. Let us first show that $\Theta_{0}^{r}$ is surjective. Given $\Phi \in \mathcal{C B}_{\hat{M}}^{\sigma, M}\left(\mathcal{B}\left(L_{2}(\mathbb{G})\right)\right)$, it is known from Proposition 3.2 that there exists $\hat{b}^{\prime} \in \hat{M}^{\prime}$ such that

$$
(\iota \otimes \Phi)(V)=V\left(\hat{b}^{\prime} \otimes 1\right)
$$

For every $f \in L_{1}(\mathbb{G})$, we let $f^{\Phi}=\left.f \circ \Phi\right|_{M} \in L_{1}(\mathbb{G})$. Then we have

$$
\rho\left(f^{\Phi}\right)=(\iota \otimes f \circ \Phi)(V)=\rho(f) \hat{b}^{\prime} .
$$

This shows that $\hat{b}^{\prime}$ is a completely bounded right multiplier of $L_{1}(\mathbb{G})$ associated with the right regular representation $\rho$ (see the discussion in Sec. 4).

Since $\mathbb{G}$ is a discrete quantum group, $L_{1}(\mathbb{G})$ has a contractive identity $f_{0}$. In this case, $\rho\left(f_{0}\right)=1$ is the identity operator in $\hat{M}^{\prime}$. We claim that $f_{0}^{\Phi}$ is an element in $L_{1}(\mathbb{G})$ such that $\Theta_{0}^{r}\left(f_{0}^{\Phi}\right)=\Phi$ on $\mathcal{B}\left(L_{2}(\mathbb{G})\right)$. 
To see this, let us consider $x=(\hat{f} \otimes \iota)(V) \in M$ for some $\hat{f} \in \hat{M}_{*}^{\prime}$. For every $\omega \in \mathcal{T}\left(L_{2}(\mathbb{G})\right.$ ), we let $f \in L_{1}(\mathbb{G})$ denote the restriction of $\omega$ to $M$. Then we have

$$
\begin{aligned}
\left\langle\Theta_{0}^{r}\left(f_{0}^{\Phi}\right)(x), \omega\right\rangle & =\left\langle f \otimes f_{0}^{\Phi}, V(x \otimes 1) V^{*}\right\rangle=\left\langle f \star f_{0}^{\Phi}, x\right\rangle \\
& =\left\langle\hat{f} \otimes f \star f_{0}^{\Phi}, V\right\rangle=\left\langle\hat{f}, \rho\left(f \star f_{0}^{\Phi}\right)\right\rangle=\left\langle\hat{f}, \rho(f) \rho\left(f_{0}^{\Phi}\right)\right\rangle \\
& =\left\langle\hat{f}, \rho(f) \rho\left(f_{0}\right) \hat{b}^{\prime}\right\rangle=\left\langle\hat{f}, \rho(f) \hat{b}^{\prime}\right\rangle=\left\langle\hat{f}, \rho\left(f^{\Phi}\right)\right\rangle \\
& =\langle\hat{f} \otimes f \circ \Phi, V\rangle=\langle\Phi(x), \omega\rangle .
\end{aligned}
$$

This shows that $\Theta_{0}^{r}\left(f_{0}^{\Phi}\right)=\Phi$ on the weak* dense subspace

$$
\hat{\rho}\left(\hat{M}_{*}^{\prime}\right)=\left\{x=(\hat{f} \otimes \iota)(V): \hat{f} \in \hat{M}_{*}^{\prime}\right\}
$$

of $M$. By the normality of $\Phi$ and $\Theta_{0}^{r}\left(f_{0}^{\Phi}\right)$, we get $\Theta_{0}^{r}\left(f_{0}^{\Phi}\right)=\Phi$ on $M$. Moreover, since both $\Theta_{0}^{r}\left(f_{0}^{\Phi}\right)$ and $\Phi$ are $\hat{M}$-bimodule maps, we have

$$
\Theta_{0}^{r}\left(f_{0}^{\Phi}\right)(x \hat{y})=\Theta_{0}^{r}\left(f_{0}^{\Phi}\right)(x) \hat{y}=\Phi(x) \hat{y}=\Phi(x \hat{y})
$$

for all $x \in M$ and $\hat{y} \in \hat{M}$. Therefore, we must have $\Theta_{0}^{r}\left(f_{0}^{\Phi}\right)=\Phi$ on $\mathcal{B}\left(L_{2}(\mathbb{G})\right)$ by Proposition 2.2. This shows that $\Theta_{0}^{r}$ is an algebra isomorphism from $L_{1}(\mathbb{G})$ onto $\mathcal{C B}_{\hat{M}}^{\sigma, M}\left(\mathcal{B}\left(L_{2}(\mathbb{G})\right)\right)$.

From this discussion, it is easy to see that if $\left[\Phi_{i j}\right] \in M_{n}\left(\mathcal{C B}_{\hat{M}}^{\sigma, M}\left(\mathcal{B}\left(L_{2}(\mathbb{G})\right)\right)\right)$, then $\left[f_{0}^{\Phi_{i j}}\right]$ is an element in $M_{n}\left(L_{1}(\mathbb{G})\right)$ with

$$
\left\|\left[f_{0}^{\Phi_{i j}}\right]\right\|_{M_{n}\left(L_{1}(\mathbb{G})\right)} \leq\left\|\left[\Phi_{i j}\right]\right\|_{M_{n}\left(\mathcal{C} \mathcal{B}_{M}^{\sigma, M}\left(\mathcal{B}\left(L_{2}(\mathbb{G})\right)\right)\right)} .
$$

Therefore, $\Theta_{0}^{r}$ is a completely isometric algebra isomorphism from $L_{1}(\mathbb{G})$ onto $\mathcal{C B}_{\hat{M}}^{\sigma, M}\left(\mathcal{B}\left(L_{2}(\mathbb{G})\right)\right)$.

We can analogously prove the following result for $\Theta_{0}^{l}$.

Theorem 3.5. Let $\mathbb{G}$ be a discrete quantum group. Then the map $\Theta_{0}^{l}$ is a completely isometric algebra anti-isomorphism from $L_{1}(\mathbb{G})$ onto $\mathcal{C B}_{\hat{M}^{\prime}}^{\sigma, M}\left(\mathcal{B}\left(L_{2}(\mathbb{G})\right)\right.$ ).

\section{Completely Bounded Right Multipliers of $L_{1}(\mathbb{G})$}

In this section, we will be concerned with generalizing Theorem 3.4 to arbitrary locally compact quantum groups. To achieve this goal, we need first to consider an appropriate extension of $\Theta_{0}^{r}$ to a larger Banach algebra (a kind of multiplier algebra of $L_{1}(\mathbb{G})$ ) because the completely contractive Banach algebra $\mathcal{C B}_{\hat{M}}^{\sigma, M}\left(\mathcal{B}\left(L_{2}(\mathbb{G})\right)\right)$ is unital and a dual space. However, the Banach algebra $L_{1}(\mathbb{G})$ is in general not a dual space, and it is unital if and only if $\mathbb{G}$ is discrete.

Let us recall from Proposition 3.2 that if $\Phi \in \mathcal{C B}_{\hat{M}}^{\sigma, M}\left(\mathcal{B}\left(L_{2}(\mathbb{G})\right)\right)$, then there exists $\hat{b}^{\prime} \in \hat{M}^{\prime}$ such that

$$
(\iota \otimes \Phi)(V)=V\left(\hat{b}^{\prime} \otimes 1\right) .
$$


It is also known from (3.5) that for every $f \in L_{1}(\mathbb{G})$, we have

$$
\rho(f) \hat{b}^{\prime}=(\iota \otimes f \circ \Phi)(V)=\rho\left(f^{\Phi}\right) .
$$

Since $\rho$ is an injective map, we obtain a completely bounded map

$$
m_{\hat{b}^{\prime}}^{r}=\left(\left.\Phi\right|_{M}\right)_{*}: f \in L_{1}(\mathbb{G}) \rightarrow \rho^{-1}\left(\rho(f) \hat{b}^{\prime}\right)=f^{\Phi} \in L_{1}(\mathbb{G})
$$

on $L_{1}(\mathbb{G})$ with $\left\|m_{\hat{b}^{\prime}}^{r}\right\|_{\mathrm{cb}} \leq\|\Phi\|_{\mathrm{cb}}$. We call such an operator $\hat{b}^{\prime} \in \hat{M}^{\prime}$ a completely bounded right multiplier of $L_{1}(\mathbb{G})$. In general, we say that an operator $\hat{b}^{\prime} \in \hat{M}^{\prime}$ is a completely bounded right multiplier of $L_{1}(\mathbb{G})$ (associated with the right fundamental unitary operator $V)$ if we have $\rho(f) \hat{b}^{\prime} \in \rho\left(L_{1}(\mathbb{G})\right)$ for all $f \in L_{1}(\mathbb{G})$ and the induced map

$$
m_{\hat{b}^{\prime}}^{r}: f \in L_{1}(\mathbb{G}) \rightarrow \rho^{-1}\left(\rho(f) \hat{b}^{\prime}\right) \in L_{1}(\mathbb{G})
$$

is completely bounded on $L_{1}(\mathbb{G})$. We let $M_{\mathrm{cb}}^{r}\left(L_{1}(\mathbb{G})\right)$ denote the space of all completely bounded right multipliers of $L_{1}(\mathbb{G})$. Then it is easy to see that $M_{\mathrm{cb}}^{r}\left(L_{1}(\mathbb{G})\right)$ is a unital subalgebra of $\hat{M}^{\prime}$ and we can identify $L_{1}(\mathbb{G})$ with the subalgebra $\rho\left(L_{1}(\mathbb{G})\right)$ in $M_{\mathrm{cb}}^{r}\left(L_{1}(\mathbb{G})\right)$.

We remark that completely bounded (left, right, and double) multipliers of $A(\mathbb{K})=L_{1}(\hat{\mathbb{K}})$ for Kac algebras $\mathbb{K}$ (associated with the left fundamental unitary operator $W$ ) have been studied by Kraus and Ruan in [14]. Some equivalent conditions for left completely bounded multipliers on $A(\mathbb{K})$ were proved in [14, Proposition 5.6]. The following proposition shows that these results can be analoguously generalized to locally compact quantum groups.

Let $\mathbb{G}$ be a locally compact quantum group, and let $\rho_{*}: L_{1}\left(\hat{\mathbb{G}}^{\prime}\right)=\hat{M}_{*}^{\prime} \rightarrow M$ be the complete contraction defined by

$$
\rho_{*}\left(\hat{f}^{\prime}\right)=\left(\hat{f}^{\prime} \otimes \iota\right)(V) .
$$

We have

$$
\left\langle\rho_{*}\left(\hat{f}^{\prime}\right), f\right\rangle=\left\langle\hat{f}^{\prime} \otimes f, V\right\rangle=\left\langle\hat{f}^{\prime}, \rho(f)\right\rangle
$$

for all $f \in L_{1}(\mathbb{G})$ and $\hat{f}^{\prime} \in L_{1}\left(\hat{\mathbb{G}}^{\prime}\right)$. It is known that

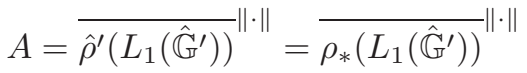

is a Hopf- $C^{*}$-subalgebra of $M$. The dual space $A^{*}$ is a completely contractive Banach algebra with mulplication given by

$$
\left\langle\tilde{f}_{1} \star \tilde{f}_{2}, \rho_{*}\left(\hat{f}^{\prime}\right)\right\rangle=\left\langle\hat{f}^{\prime} \otimes \tilde{f}_{1} \otimes \tilde{f}_{2}, V_{23} V_{12} V_{23}^{*}\right\rangle=\left\langle\hat{f}^{\prime} \otimes \tilde{f}_{1} \otimes \tilde{f}_{2}, V_{12} V_{13}\right\rangle
$$

and $A^{*}$ contains $L_{1}(\mathbb{G})$ as a norm closed two-sided ideal.

Proposition 4.1. Let $\hat{b}^{\prime}$ be an operator in $\hat{M}^{\prime}$. Then the following are equivalent:

(1) $\hat{b}^{\prime} \in M_{\mathrm{cb}}^{r}\left(L_{1}(\mathbb{G})\right)$ satisfies $\left\|m_{\hat{b}^{\prime}}^{r}\right\|_{\mathrm{cb}} \leq 1$;

(2) we have

$$
\left\|\left[\rho_{*}\left(\hat{b}^{\prime} \cdot \hat{f}_{i j}^{\prime}\right)\right]\right\| \leq\left\|\left[\rho_{*}\left(\hat{f}_{i j}^{\prime}\right)\right]\right\|
$$

for all $\left[\hat{f}_{i j}^{\prime}\right] \in M_{n}\left(L_{1}\left(\hat{\mathbb{G}}^{\prime}\right)\right)$ and $n \in \mathbb{N}$; 
$\left(2^{\prime}\right)$ there exists a complete contraction $M_{\hat{b}^{\prime}}^{r}$ on A such that

$$
M_{\hat{b}^{\prime}}^{r}\left(\rho_{*}\left(\hat{f}^{\prime}\right)\right)=\rho_{*}\left(\hat{b}^{\prime} \cdot \hat{f}^{\prime}\right)
$$

for all $\hat{f}^{\prime} \in L_{1}\left(\hat{\mathbb{G}}^{\prime}\right)$

(3) there exists a complete contraction $\tilde{m}_{\hat{b}^{\prime}}^{r}$ on the Banach algebra $A^{*}$ such that

$$
\tilde{m}_{\hat{b}^{\prime}}^{r}(f \star \tilde{f})=f \star \tilde{m}_{\hat{b}^{\prime}}^{r}(\tilde{f})
$$

for all $f \in L_{1}(\mathbb{G})$ and $\tilde{f} \in A^{*}$ and $\left(\tilde{m}_{\hat{b}^{\prime}}^{r}\right)_{\mid L_{1}(\mathbb{G})}=m_{\hat{b}^{\prime}}^{r}$;

(4) there exists a normal complete contraction $\Phi_{\hat{b}^{\prime}}^{r}$ on $M$ such that

$$
\left(\iota \otimes \Phi_{\hat{b}^{\prime}}^{r}\right)(V)=V\left(\hat{b}^{\prime} \otimes 1\right) .
$$

In this case, we actually have $M_{\hat{b}^{\prime}}^{r}=\left(m_{\hat{b}^{\prime}}^{r}\right)_{\mid A}^{*}$.

Proof. Assume that we have (1). For any $\left[f_{k l}\right] \in M_{n}\left(L_{1}(\mathbb{G})\right)$, we have

$$
\begin{aligned}
\left\|\left[\left\langle\rho_{*}\left(\hat{b}^{\prime} \cdot \hat{f}_{i j}^{\prime}\right), f_{k l}\right\rangle\right]\right\| & =\left\|\left[\left\langle\hat{f}_{i j}^{\prime}, \rho\left(f_{k l}\right) \hat{b}^{\prime}\right\rangle\right]\right\|=\left\|\left[\left\langle\rho_{*}\left(\hat{f}_{i j}^{\prime}\right), m_{\hat{b}^{\prime}}^{r}\left(f_{k l}\right)\right\rangle\right]\right\| \\
& \leq\left\|\left[\rho_{*}\left(\hat{f}_{i j}^{\prime}\right)\right]\right\|\left\|\left[f_{k l}\right]\right\| .
\end{aligned}
$$

We obtain (2) by taking the supremum over all $\left[f_{k l}\right]$ in the unit ball of $M_{n}\left(L_{1}(\mathbb{G})\right.$ ).

It is clear that (2) is equivalent to $\left(2^{\prime}\right)$ with $M_{\hat{b}^{\prime}}^{r}: A \rightarrow A$ determined by

$$
M_{\hat{b}^{\prime}}^{r}\left(\rho_{*}\left(\hat{f}^{\prime}\right)\right)=\rho_{*}\left(\hat{b}^{\prime} \cdot \hat{f}^{\prime}\right) .
$$

Now if we have $\left(2^{\prime}\right)$, we let $\tilde{m}_{\hat{b}^{\prime}}^{r}=\left(M_{\hat{b}^{\prime}}^{r}\right)^{*}$ be the adjoint map on $A^{*}$. For any $\tilde{f} \in A^{*}$ and $\hat{f}^{\prime} \in L_{1}\left(\hat{\mathbb{G}}^{\prime}\right)$, we obtain

$$
\left\langle\tilde{m}_{\hat{b}^{\prime}}^{r}(\tilde{f}), \rho_{*}\left(\hat{f}^{\prime}\right)\right\rangle=\left\langle\tilde{f}, M_{\hat{b}^{\prime}}^{r}\left(\rho_{*}\left(\hat{f}^{\prime}\right)\right)\right\rangle=\left\langle\tilde{f}, \rho_{*}\left(\hat{b}^{\prime} \cdot \hat{f}^{\prime}\right)\right\rangle .
$$

This is equivalent to

$$
\left\langle\hat{f}^{\prime} \otimes \tilde{m}_{\hat{b}^{\prime}}^{r}(\tilde{f}), V\right\rangle=\left\langle\hat{f}^{\prime} \otimes \tilde{f}, V\left(\hat{b}^{\prime} \otimes 1\right)\right\rangle .
$$

Since $L_{1}(\mathbb{G})$ is a norm closed two-sided ideal in $A^{*}$, we have

$$
\begin{aligned}
\left\langle\tilde{m}_{\hat{b}^{\prime}}^{r}(f \star \tilde{f}), \rho_{*}\left(\hat{f}^{\prime}\right)\right\rangle & =\left\langle\hat{f}^{\prime} \otimes f \star \tilde{f}, V\left(\hat{b}^{\prime} \otimes 1\right)\right\rangle=\left\langle\hat{f}^{\prime} \otimes f \otimes \tilde{f}, V_{23} V_{12} V_{23}^{*}\left(\hat{b}^{\prime} \otimes 1\right)\right\rangle \\
& =\left\langle\hat{f}^{\prime} \otimes f \otimes \tilde{f}, V_{12} V_{13}\left(\hat{b}^{\prime} \otimes 1\right)\right\rangle=\left\langle\hat{f}^{\prime} \otimes f \otimes \tilde{m}_{\hat{b}^{\prime}}^{r}(\tilde{f}), V_{12} V_{13}\right\rangle \\
& =\left\langle\hat{f}^{\prime} \otimes f \star \tilde{m}_{\hat{b}^{\prime}}^{r}(\tilde{f}), V\right\rangle=\left\langle f \star \tilde{m}_{\hat{b}^{\prime}}^{r}(\tilde{f}), \rho_{*}\left(\hat{f}^{\prime}\right)\right\rangle
\end{aligned}
$$

for all $f \in L_{1}(\mathbb{G}), \tilde{f} \in A^{*}$ and $\hat{f}^{\prime} \in L_{1}\left(\hat{\mathbb{G}}^{\prime}\right)$. This shows that

$$
\tilde{m}_{\hat{b}^{\prime}}^{r}(f \star \tilde{f})=f \star \tilde{m}_{\hat{b}^{\prime}}^{r}(\tilde{f}) \in L_{1}(\mathbb{G}) .
$$

Since the multiplication $m$ is a complete quotient map from $L_{1}(\mathbb{G}) \hat{\otimes} L_{1}(\mathbb{G})$ onto $L_{1}(\mathbb{G})$, we can conclude that $\tilde{m}_{\hat{b}^{\prime}}^{r}$ maps $L_{1}(\mathbb{G})$ into $L_{1}(\mathbb{G})$. Moreover, the restriction 
of $\tilde{m}_{\hat{b}^{\prime}}^{r}$ to $L_{1}(\mathbb{G})$ is equal to $m_{\hat{b}^{\prime}}^{r}$ since

$$
\left\langle\tilde{m}_{\hat{b}^{\prime}}^{r}(f), \rho_{*}\left(\hat{f}^{\prime}\right)\right\rangle=\left\langle f, \rho_{*}\left(\hat{b}^{\prime} \cdot \hat{f}^{\prime}\right)\right\rangle=\left\langle\rho(f) \hat{b}^{\prime}, \hat{f}^{\prime}\right\rangle=\left\langle m_{\hat{b}^{\prime}}^{r}(f), \rho_{*}\left(\hat{f}^{\prime}\right)\right\rangle .
$$

This proves (3). It is clear that (3) implies (1).

Now if we have $(3)$, it is easy to see that $\Phi_{\hat{b}^{\prime}}^{r}=\left(m_{\hat{b}^{\prime}}^{r}\right)^{*}$ is a normal complete contraction on $M$ such that

$$
\left\langle\hat{f}^{\prime} \otimes f,\left(\iota \otimes \Phi_{\hat{b}^{\prime}}^{r}\right)(V)\right\rangle=\left\langle\hat{f}^{\prime} \otimes m_{\hat{b}^{\prime}}^{r}(f), V\right\rangle=\left\langle\hat{f}^{\prime} \otimes f, V\left(\hat{b}^{\prime} \otimes 1\right)\right\rangle .
$$

This proves (4).

Finally, let us assume (4). Then for any $f \in L_{1}(\mathbb{G})$, we have

$$
\rho(f) \hat{b}^{\prime}=(\iota \otimes f)\left(V\left(\hat{b}^{\prime} \otimes 1\right)\right)=\left(\iota \otimes f \circ \Phi_{\hat{b}^{\prime}}^{r}\right)(V)=\rho\left(\left(\Phi_{\hat{b}^{\prime}}^{r}\right)_{*}(f)\right) \in \rho\left(L_{1}(\mathbb{G})\right)
$$

and $m_{\hat{b}^{\prime}}^{r}=\left(\Phi_{\hat{b}^{\prime}}^{r}\right)_{*}$ is a complete contraction on $L_{1}(\mathbb{G})$. This shows that $\hat{b}^{\prime}$ is a completely bounded right multiplier on $L_{1}(\mathbb{G})$, and we obtain $(1)$.

Following Johnson's terminology in [12], a completely bounded map $T$ on $L_{1}(\mathbb{G})$ is said to be a completely bounded right centralizer on $L_{1}(\mathbb{G})$ if it satisfies

$$
f_{1} \star T\left(f_{2}\right)=T\left(f_{1} \star f_{2}\right)
$$

for all $f_{1}, f_{2} \in L_{1}(\mathbb{G})$. We let $C_{\mathrm{cb}}^{r}\left(L_{1}(\mathbb{G})\right)$ denote the space of all completely bounded right centralizers of $L_{1}(\mathbb{G})$. Since $L_{1}(\mathbb{G})$ is a closed two-sided ideal in $A^{*}$, it is easy to see that for every $\tilde{f} \in A^{*}$, the right multiplication map

$$
m_{\tilde{f}}^{r}: f \in L_{1}(\mathbb{G}) \rightarrow f \star \tilde{f} \in L_{1}(\mathbb{G})
$$

is a completely bounded right centralizer of $L_{1}(\mathbb{G})$. This determines an injective completely contractive anti-homomorphism from $L_{1}(\mathbb{G}) \subseteq A^{*}$ into $C_{\mathrm{cb}}^{r}\left(L_{1}(\mathbb{G})\right)$.

It is also known from (3) of Proposition 4.1 that if $\hat{b}^{\prime}$ is a completely bounded right multiplier of $L_{1}(\mathbb{G})$, then the induced map $m_{\hat{b}^{\prime}}^{r}$ is a completely bounded right centralizer of $L_{1}(\mathbb{G})$. We have a canonical linear injection

$$
m^{r}: \hat{b}^{\prime} \in M_{\mathrm{cb}}^{r}\left(L_{1}(\mathbb{G})\right) \rightarrow m_{\hat{b}^{\prime}}^{r} \in C_{\mathrm{cb}}^{r}\left(L_{1}(\mathbb{G})\right),
$$

which is an anti-homomorphism since

$$
m_{\hat{b}_{1}^{\prime} \hat{b}_{2}^{\prime}}^{r}(f)=\rho^{-1}\left(\rho(f) \hat{b}_{1}^{\prime} \hat{b}_{2}^{\prime}\right)=\rho^{-1}\left(\rho\left(m_{\hat{b}_{1}^{\prime}}^{r}(f)\right) \hat{b}_{2}^{\prime}\right)=m_{\hat{b}_{2}^{\prime}}^{r} \circ m_{\hat{b}_{1}^{\prime}}^{r}(f) .
$$

In particular, if $\hat{b}^{\prime}=\rho(f)$ for some $f \in L_{1}(\mathbb{G})$, then we actually have

$$
m_{\rho(f)}^{r}=m_{f}^{r} .
$$

If we consider the adjoint maps $\Phi_{\hat{b}^{\prime}}^{r}=\left(m_{\hat{b}^{\prime}}^{r}\right)^{*}$, we obtain an injective algebraic homomorphism

$$
\Phi^{r}: \hat{b}^{\prime} \in M_{\mathrm{cb}}^{r}\left(L_{1}(\mathbb{G})\right) \rightarrow \Phi_{\hat{b}^{\prime}}^{r} \in \mathcal{C B}^{\sigma}\left(L_{\infty}(\mathbb{G})\right) .
$$


We say that a normal completely bounded map $\Phi$ on $L_{\infty}(\mathbb{G})$ is right covariant if it satisfies

$$
(\iota \otimes \Phi) \circ \Gamma=\Gamma \circ \Phi .
$$

We let $\mathcal{C B}_{\text {cov }}^{\sigma}\left(L_{\infty}(\mathbb{G})\right)$ denote the space of all completely bounded normal right covariant maps on $L_{\infty}(\mathbb{G})$. We note that a normal completely bounded map $\Phi$ on $L_{\infty}(\mathbb{G})$ satisfies condition $(4.6)$ if and only if it is a right $L_{1}(\mathbb{G})$-module map on $L_{\infty}(\mathbb{G})$. Therefore, we may also use the notation $\mathcal{B}_{L_{1}(\mathbb{G})^{r}}^{\sigma}\left(L_{\infty}(\mathbb{G})\right)$ for $\mathcal{C B}_{\text {cov }}^{\sigma}\left(L_{\infty}(\mathbb{G})\right)$.

It is easy to see that right covariance is a dual characterization of the right centralizer property, i.e. (4.6) is just the dual form of condition (4.4) since

$$
\left\langle\left(\iota \otimes \Phi_{T}\right) \circ \Gamma(x), f \otimes g\right\rangle=\langle x, f \star T(g)\rangle
$$

and

$$
\left\langle\Gamma \circ \Phi_{T}(x), f \otimes g\right\rangle=\langle x, T(f \star g)\rangle
$$

for all $x \in L_{\infty}(\mathbb{G})$ and $f, g \in L_{1}(\mathbb{G})$. Therefore, the adjoint map

$$
T \in C_{\mathrm{cb}}^{r}\left(L_{1}(\mathbb{G})\right) \rightarrow \Phi_{T}=T^{*} \in \mathcal{C B}_{\mathrm{cov}}^{\sigma}\left(L_{\infty}(\mathbb{G})\right)
$$

is a completely isometric and algebraic anti-isomorphism from $C_{\mathrm{cb}}^{r}\left(L_{1}(\mathbb{G})\right)$ onto $\mathcal{C B}_{\text {cov }}^{\sigma}\left(L_{\infty}(\mathbb{G})\right)$. We can summarize our discussion in the following result.

Proposition 4.2. If we define the operator space matrix norm

$$
\left\|\left[\hat{b}_{i j}^{\prime}\right]\right\|_{M_{n}\left(M_{\mathrm{cb}}^{r}\left(L_{1}(\mathbb{G})\right)\right)}=\left\|\left[m_{\hat{b}_{i j}^{\prime}}^{r}\right]\right\|_{\mathrm{cb}}=\left\|\left[\Phi_{\hat{b}_{i j}^{\prime}}^{r}\right]\right\|_{\mathrm{cb}}
$$

on $M_{\mathrm{cb}}^{r}\left(L_{1}(\mathbb{G})\right)$, then

$$
m^{r}: \hat{b}^{\prime} \in M_{\mathrm{cb}}^{r}\left(L_{1}(\mathbb{G})\right) \rightarrow m_{\hat{b}^{\prime}}^{r} \in C_{\mathrm{cb}}^{r}\left(L_{1}(\mathbb{G})\right)
$$

is a completely isometric algebra anti-homomorphism, and

$$
\Phi^{r}: \hat{b}^{\prime} \in M_{\mathrm{cb}}^{r}\left(L_{1}(\mathbb{G})\right) \rightarrow \Phi_{\hat{b}^{\prime}}^{r} \in \mathcal{C B}_{\mathrm{cov}}^{\sigma}\left(L_{\infty}(\mathbb{G})\right)
$$

is a completely isometric algebra homomorphism.

We can show in the following representation theorem that $m^{r}$ and $\Phi^{r}$ are actually surjective. Therefore, we may regard $M_{\mathrm{cb}}^{r}\left(L_{1}(\mathbb{G})\right)$ as a concrete realization of $C_{\mathrm{cb}}^{r}\left(L_{1}(\mathbb{G})\right)$ or $\mathcal{C B}_{\mathrm{cov}}^{\sigma}\left(L_{\infty}(\mathbb{G})\right)$ inside the von Neumann algebra $\hat{M}^{\prime}$ (see the detailed discussion for Kac algebras in [14]). Now let us assume that $T \in C_{\mathrm{cb}}^{r}\left(L_{1}(\mathbb{G})\right)$ is a completely bounded right centralizer and $\Phi_{T}$ is the corresponding right covariant map in $\mathcal{C B}_{\text {cov }}^{\sigma}\left(L_{\infty}(\mathbb{G})\right)$. We can obtain the following result.

Proposition 4.3. For each $\Phi_{T} \in \mathcal{C B}_{\text {cov }}^{\sigma}\left(L_{\infty}(\mathbb{G})\right)$, there exists a unique map $\tilde{\Phi}_{T} \in$ $\mathcal{C B}_{\hat{M}}^{\sigma, M}\left(\mathcal{B}\left(L_{2}(\mathbb{G})\right)\right)$ such that $\left.\tilde{\Phi}_{T}\right|_{L_{\infty}(\mathbb{G})}=\Phi_{T}$. This defines a completely isometric algebra homomorphism

$$
\mathcal{E}: \Phi_{T} \in \mathcal{C B}_{\text {cov }}^{\sigma}\left(L_{\infty}(\mathbb{G})\right) \rightarrow \tilde{\Phi}_{T} \in \mathcal{C B}_{\hat{M}}^{\sigma, M}\left(\mathcal{B}\left(L_{2}(\mathbb{G})\right)\right) .
$$


Proof. Our first aim is to extend $\Phi_{T}$ to a map $\tilde{\Phi}_{T} \in \mathcal{C B}_{\hat{M}}^{\sigma, M}\left(\mathcal{B}\left(L_{2}(\mathbb{G})\right)\right)$. To this end, let us consider the map

$$
\Psi: x \in \mathcal{B}\left(L_{2}(\mathbb{G})\right) \rightarrow V^{*}\left[\left(\iota \otimes \Phi_{T}\right)\left(V(x \otimes 1) V^{*}\right)\right] V \in \mathcal{B}\left(L_{2}(\mathbb{G})\right) \bar{\otimes} M .
$$

We claim that the range of $\Psi$ is actually contained in $\mathcal{B}\left(L_{2}(\mathbb{G})\right) \bar{\otimes} \mathbb{C}$. To see this, first note that for $x \in M$ and $\hat{x} \in \hat{M}$, we have

$$
\begin{aligned}
\Psi(x \hat{x}) & =V^{*}\left[\left(\iota \otimes \Phi_{T}\right)\left(V(x \hat{x} \otimes 1) V^{*}\right)\right] V=V^{*}\left[\left(\iota \otimes \Phi_{T}\right)(\Gamma(x))\right] V(\hat{x} \otimes 1) \\
& =V^{*} V\left(\Phi_{T}(x) \otimes 1\right) V^{*} V(\hat{x} \otimes 1)=\Phi_{T}(x) \hat{x} \otimes 1 .
\end{aligned}
$$

This shows that

$$
\Psi(x \hat{x})=\Phi_{T}(x) \hat{x} \otimes 1
$$

Similarly we obtain:

$$
\Psi(\hat{x} x)=\hat{x} \Phi_{T}(x) \otimes 1
$$

(In particular, if we let $x=1$, we get $\Phi_{T}(1) \in \hat{M}^{\prime} \cap M=\mathbb{C} 1$.) Using the normality of $\Psi$ and the density result from Proposition 2.2, we can conclude that $\Psi(x) \in$ $\mathcal{B}\left(L_{2}(\mathbb{G})\right) \bar{\otimes} \mathbb{C}$ for all $x \in \mathcal{B}\left(L_{2}(\mathbb{G})\right)$.

Let $\tilde{\Phi}_{T}$ be the normal map on $\mathcal{B}\left(L_{2}(\mathbb{G})\right)$ such that

$$
\tilde{\Phi}_{T}(x) \otimes 1=V^{*}\left[\left(\iota \otimes \Phi_{T}\right)\left(V(x \otimes 1) V^{*}\right)\right] V .
$$

Then it is clear that $\left.\tilde{\Phi}_{T}\right|_{L_{\infty}(\mathbb{G})}=\Phi_{T}$ and $\tilde{\Phi}_{T}$ is an $\hat{M}$-bimodule map. Therefore, $\tilde{\Phi}_{T} \in \mathcal{C B}_{\hat{M}}^{\sigma, M}\left(\mathcal{B}\left(L_{2}(\mathbb{G})\right)\right)$ such that

$$
\left\|\Phi_{T}\right\|_{\mathrm{cb}} \leq\left\|\tilde{\Phi}_{T}\right\|_{\mathrm{cb}} \leq\left\|\Phi_{T}\right\|_{\mathrm{cb}}=\|T\|_{\mathrm{cb}} .
$$

The uniqueness of $\tilde{\Phi}_{T}$ is obvious. This shows that $\mathcal{E}$ is an isometric linear injection. It is easy to see that $\mathcal{E}$ is actually a complete isometry and is an algebra homomorphism.

Corollary 4.4. For each $T \in C_{\mathrm{cb}}^{r}\left(L_{1}(\mathbb{G})\right)$, there exists a unique $\hat{b}^{\prime} \in M_{\mathrm{cb}}^{r}\left(L_{1}(\mathbb{G})\right)$ such that

$$
T=m_{\hat{b}^{\prime}}^{r} \quad \text { and } \quad \Phi_{T}=\Phi_{\hat{b}^{\prime}}^{r} .
$$

Thus, $M_{\mathrm{cb}}^{r}\left(L_{1}(\mathbb{G})\right)$ is completely isometric and algebra anti-isomorphic (respectively, completely isometric and algebra isomorphic) to $C_{\mathrm{cb}}^{r}\left(L_{1}(\mathbb{G})\right.$ ) (respectively, $\left.\mathcal{C B}_{\operatorname{cov}}^{\sigma}\left(L_{\infty}(\mathbb{G})\right)\right)$

Proof. We have already shown that

$$
\Phi^{r}: \hat{b}^{\prime} \in M_{\mathrm{cb}}^{r}\left(L_{1}(\mathbb{G})\right) \rightarrow \Phi_{\hat{b}^{\prime}}^{r} \in \mathcal{C B}_{\mathrm{cov}}^{\sigma}\left(L_{\infty}(\mathbb{G})\right)
$$


is a completely isometric algebra homomorphism. We only need to prove that this map is also onto. Given $T \in C_{\mathrm{cb}}^{r}\left(L_{1}(\mathbb{G})\right)$, let $\tilde{\Phi}_{T}$ be the corresponding map contained in $\mathcal{C B}_{\hat{M}}^{\sigma, M}\left(\mathcal{B}\left(L_{2}(\mathbb{G})\right)\right)$ such that $\left.\tilde{\Phi}_{T}\right|_{M}=\Phi_{T}$. Then there exists $\hat{b}^{\prime} \in \hat{M}^{\prime}$ such that

$$
\left(\iota \otimes \tilde{\Phi}_{T}\right)(V)=V\left(\hat{b}^{\prime} \otimes 1\right) .
$$

This implies that $T=m_{\hat{b}^{\prime}}^{r}(f)$ and thus $\Phi_{T}=\Phi_{\hat{b}^{\prime}}^{r}$.

Corollary 4.4 shows that we can obtain a completely contractive unital antihomomorphism

$$
\tilde{\rho}: T \in C_{\mathrm{cb}}^{r}\left(L_{1}(\mathbb{G})\right) \rightarrow \tilde{\rho}(T)=\hat{b}^{\prime} \in \hat{M}^{\prime}
$$

such that $T=m_{\hat{b}^{\prime}}^{r}$. This proivdes a natural extension of $\rho$ to $C_{\mathrm{cb}}^{r}\left(L_{1}(\mathbb{G})\right)$. Indeed, we can (anti-isomorphically) identify $L_{1}(\mathbb{G})$ with the subalgebra $m^{r}\left(L_{1}(\mathbb{G})\right)=\left\{m_{f}^{r}\right.$ : $\left.f \in L_{1}(\mathbb{G})\right\}$ in $C_{\mathrm{cb}}^{r}\left(L_{1}(\mathbb{G})\right)$ and we can conclude from (4.5) and Corollary 4.4 that $\tilde{\rho}\left(m_{f}^{r}\right)=\rho(f)$ for $f \in L_{1}(\mathbb{G})$. This generalizes the classical result (cf. [31, 14]) to locally compact quantum groups. In particular, $L_{1}(\mathbb{G})$ is unital, i.e. $\mathbb{G}$ is a discrete quantum group, if and only if we have $L_{1}(\mathbb{G}) \cong C_{\mathrm{cb}}^{r}\left(L_{1}(\mathbb{G})\right)$, or equivalently, we have

$$
M_{\mathrm{cb}}^{r}\left(L_{1}(\mathbb{G})\right)=\rho\left(L_{1}(\mathbb{G})\right) .
$$

We may also (anti-isomorphically) identify $A^{*}$ with a subalgebra $\tilde{m}^{r}\left(A^{*}\right)$ in $C_{\mathrm{cb}}^{r}\left(L_{1}(\mathbb{G})\right)$. Then $\tilde{\rho}$ defines a completely contractive homomorphism from $A^{*}$ into $M_{\mathrm{cb}}^{r}\left(L_{1}(\mathbb{G})\right) \subseteq \hat{M}^{\prime}$, which extends $\rho$. In general, $\tilde{\rho}\left(A^{*}\right)$ is not equal to $M_{\mathrm{cb}}^{r}\left(L_{1}(\mathbb{G})\right)$. We have the equality if and only if $\mathbb{G}$ is co-amenable. We will pursue a thorough investigation of the co-amenable case in the forthcoming paper [21].

Summarizing, we can now state the main representation theorem of this paper, which extends Theorem 3.4 to the setting of arbitrary locally compact quantum groups.

Theorem 4.5. Let $\mathbb{G}$ be a locally compact quantum group. Then there exists a completely isometric algebra isomorphism $\Theta^{r}=\mathcal{E} \circ \Phi^{r}$ from $M_{\mathrm{cb}}^{r}\left(L_{1}(\mathbb{G})\right)$ onto $\mathcal{C B}_{\hat{M}}^{\sigma, M}\left(\mathcal{B}\left(L_{2}(\mathbb{G})\right)\right)$, and we can completely isometrically and algebraically identify the following completely contractive Banach algebras

$$
M_{\mathrm{cb}}^{r}\left(L_{1}(\mathbb{G})\right) \cong \mathcal{C B}_{\mathrm{cov}}^{\sigma}\left(L_{\infty}(\mathbb{G})\right) \cong \mathcal{C B}_{\hat{M}}^{\sigma, M}\left(\mathcal{B}\left(L_{2}(\mathbb{G})\right)\right)
$$

Remark 4.6. It is known (cf. [22]) that if $G$ is a locally compact group, then the completely isometric representations $\Theta^{r}: M(G) \cong \mathcal{C B}_{L(G)}^{\sigma, L_{\infty}(G)}\left(\mathcal{B}\left(L_{2}(G)\right)\right)$ and $\hat{\Theta}: M_{\mathrm{cb}} A(G) \cong \mathcal{C B}_{L_{\infty}(G)}^{\sigma, L(G)}\left(\mathcal{B}\left(L_{2}(G)\right)\right)$ are weak*-weak* continuous (with respect to their canonical preduals). In the forthcoming paper [21], we can show that for an arbitrary locally compact quantum group $\mathbb{G}, M_{\mathrm{cb}}^{r}\left(L_{1}(\mathbb{G})\right)$ is a dual space (in fact, a dual Banach algebra) and, when $\mathbb{G}$ is co-amenable, the representation map $\Theta^{r}: M_{\mathrm{cb}}^{r}\left(L_{1}(\mathbb{G})\right) \cong \mathcal{C B}_{\hat{M}}^{\sigma, M}\left(\mathcal{B}\left(L_{2}(\mathbb{G})\right)\right)$ is weak ${ }^{*}$-weak* continuous. However, we do not know whether this is still true for general (non-co-amenable) $\mathbb{G}$. 
The difficulty lies in the fact that the passage through $\mathcal{C B}_{\text {cov }}^{\sigma}\left(L_{\infty}(\mathbb{G})\right)$ is, in general, discontinuous. This can be seen as follows. Consider the completely isometrically isomorphic spaces $X:=\mathcal{C B}_{\hat{M}}^{\sigma, M}\left(\mathcal{B}\left(L_{2}(\mathbb{G})\right)\right), Y:=\mathcal{C B}_{\text {cov }}^{\sigma}\left(L_{\infty}(\mathbb{G})\right)$ and $Z:=M_{\mathrm{cb}}^{r}\left(L_{1}(\mathbb{G})\right)$. Denote by $R$ the restriction map from $X$ to $Y$ and by $S$ the canonical map from $Y$ to $Z$. Then $\Theta^{r}$ is the inverse of $S \circ R$. Endow $X$ and $Z$ with their weak* topologies and $Y$ with the (natural) point-weak* topology. Then $R$ and $S$ can be both discontinuous. Indeed, let $G$ be a non-compact, locally compact, amenable group and $M=L_{\infty}(G)$. Let $m$ be an invariant mean on $\operatorname{LUC}(G)$, the space of bounded left uniformly continuous functions on $G$ and choose a net of measures $\mu_{i}$ in $M(G)$ which tends weak* in $\operatorname{LUC}(G)^{*}$ to $m$. Then $\mu_{i}$ tends weak* in $Z=M(G)$ to 0 (since $m$ vanishes on $C_{0}(G)$ ). Also, $\Theta^{r}\left(\mu_{i}\right)$ tends weak* in $X$ to 0 , since the weak* topology of $X$ is the one inherited from

$$
\mathcal{C B}^{\sigma}\left(\mathcal{B}\left(L_{2}(G)\right)\right)=\mathcal{C B}\left(\mathcal{K}\left(L_{2}(G)\right), \mathcal{B}\left(L_{2}(G)\right)\right)=\left(\mathcal{K}\left(L_{2}(G)\right) \hat{\otimes} \mathcal{T}\left(L_{2}(G)\right)\right)^{*},
$$

where $\mathcal{K}\left(L_{2}(G)\right)$ denotes the compact operators, and $\left\langle\Theta^{r}\left(\mu_{i}\right), K \otimes \rho\right\rangle \rightarrow 0$ for any $K \in \mathcal{K}\left(L_{2}(G)\right)$ and $\rho \in \mathcal{T}\left(L_{2}(G)\right.$ ) (again noting that $m$ vanishes on $C_{0}(G)$ ). But the restriction $R\left(\Theta^{r}\left(\mu_{i}\right)\right)$ does not tend point-weak* to 0 in $Y$ since, for example, $R\left(\Theta^{r}\left(\mu_{i}\right)\right)(1)$ converges to 1 in the weak* topology.

As an application of Theorem 4.5, we may obtain the following quantum group analogue of Wendel's result [31].

Theorem 4.7. Let $\mathbb{G}$ be a locally compact quantum group and let $T$ be a complete contraction in $C_{\mathrm{cb}}^{r}\left(L_{1}(\mathbb{G})\right)$. Then the following are equivalent:

(1) $T$ is a completely isometric linear isomorphism on $L_{1}(\mathbb{G})$;

(2) $T$ has a completely contractive inverse in $C_{\mathrm{cb}}^{r}\left(L_{1}(\mathbb{G})\right)$;

(3) there exist a unitary operator $u \in \hat{M}^{\prime}$ and a complex number $\alpha$ with $|\alpha|=1$ such that $\tilde{\Phi}_{T}(x)=\alpha u x u^{*}$ for all $x \in \mathcal{B}\left(L_{2}(\mathbb{G})\right)$.

If, in addition, $T$ is completely positive, then so is $T^{-1}$. In this case, we have $\tilde{\Phi}_{T}(x)=u x u^{*}$ for some $u \in \hat{M}^{\prime}$.

Proof. Let us first assume (1). Then $T^{-1}$ is a complete isometry on $L_{1}(\mathbb{G})$. For any $f, g \in L_{1}(\mathbb{G})$, there exists $\tilde{g} \in L_{1}(\mathbb{G})$ such that $g=T(\tilde{g})$ and thus

$$
T^{-1}(f \star g)=T^{-1}(f \star T(\tilde{g}))=T^{-1}(T(f \star \tilde{g}))=f \star T^{-1}(g) .
$$

This shows that $T^{-1}$ is a completely contractive right centralizer contained in $C_{\mathrm{cb}}^{r}\left(L_{1}(\mathbb{G})\right)$. This proves $(2)$.

If we have (2), then $T$ has a completely contractive inverse $T^{-1}$ in $C_{\mathrm{cb}}^{r}\left(L_{1}(\mathbb{G})\right)$. We can conclude from Theorem 4.5 that the corresponding map $\tilde{\Phi}_{T}=\mathcal{E}\left(\Phi_{T}\right)$ is a completely isometric linear isomorphism on $\mathcal{B}\left(L_{2}(\mathbb{G})\right)$. It now follows from the completely isometric version of Kadison's non-commutative Banach-Stone theorem (for instance, see $\left[3\right.$, Theorem 1.1]) that there exists a unitary $v \in \mathcal{B}\left(L_{2}(\mathbb{G})\right.$ ) and a ${ }^{*}$-isomorphism $\pi$ on $\mathcal{B}\left(L_{2}(\mathbb{G})\right)$ such that $\tilde{\Phi}_{T}(x)=v \pi(x)$ for all $x \in \mathcal{B}\left(L_{2}(\mathbb{G})\right)$. By 
[26, Proposition 2.9.31], $\pi$ is spatial, i.e. $\pi=\operatorname{Ad} u$ for some unitary $u \in \mathcal{B}\left(L_{2}(\mathbb{G})\right)$, so that we have

$$
\tilde{\Phi}_{T}(x)=v u x u^{*}
$$

for all $x \in \mathcal{B}\left(L_{2}(\mathbb{G})\right)$. Since $\tilde{\Phi}_{T} \in \mathcal{C B}_{\hat{M}}^{\sigma, M}\left(\mathcal{B}\left(L_{2}(\mathbb{G})\right)\right)$, we can conclude that $v=$ $\tilde{\Phi}_{T}(1) \in M \cap \hat{M}^{\prime}=\mathbb{C} 1$. Therefore, $v=\alpha 1$ with $|\alpha|=1$. Finally since $\tilde{\Phi}_{T}$ is an $\hat{M}$-bimodule map, we can conlcude that $u \in \hat{M}^{\prime}$. This proves (3).

$(3) \Rightarrow(1)$ is obvious.

Remark 4.8. We wish to point out that the covariance condition (4.6) has played a crucial role in the investigation of the intrinsic group of a Kac algebra (cf. De Cannière's work [4]). Indeed, [4, Theorem 2.3], one of the main results of the paper, shows that the covariance property can be used to characterize precisely the elements of the intrinsic group of the dual Kac algebra among its unitaries. The corresponding result for the intrinsic group of a locally compact quantum group, as well as various other intriguing characterizations and properties of the latter in terms of completely bounded multipliers, have been established very recently in [13], using results from the present work (such as our Theorem 4.7 above).

Recall that we can identify $L_{1}(\mathbb{G})$ with a subalgebra $\rho\left(L_{1}(\mathbb{G})\right)$ in $M_{\mathrm{cb}}^{r}\left(L_{1}(\mathbb{G})\right)$ such that $m_{\rho(f)}^{r}=m_{f}^{r}$ by (4.5). The next result shows that $\Theta^{r}(\rho(f))=\Theta_{0}^{r}(f)$. Therefore, we can regard $\Theta^{r}$ as a natural extension of $\Theta_{0}^{r}$.

Proposition 4.9. If $f \in L_{1}(\mathbb{G})$, then we have

$$
\Theta^{r}(\rho(f))(x)=(\iota \otimes f) V(x \otimes 1) V^{*}=\Theta_{0}^{r}(f)(x)
$$

for all $x \in \mathcal{B}\left(L_{2}(\mathbb{G})\right)$.

Proof. We first note that $\Phi_{\rho(f)}^{r}(x)=\Theta_{0}^{r}(f)(x)$ for every $x \in M$ since

$$
\begin{aligned}
\left\langle g, \Theta_{0}^{r}(f)(x)\right\rangle & =\left\langle g \otimes f, V(x \otimes 1) V^{*}\right\rangle=\langle g \star f, x\rangle \\
& =\left\langle m_{f}^{r}(g), x\right\rangle=\left\langle m_{\rho(f)}^{r}(g), x\right\rangle=\left\langle g, \Phi_{\rho(f)}^{r}(x)\right\rangle
\end{aligned}
$$

for all $g \in L_{1}(\mathbb{G})$. Now for $x \in \mathcal{B}\left(L_{2}(\mathbb{G})\right)$, we obtain

$$
\begin{aligned}
V\left(\Theta^{r}(\rho(f))(x) \otimes 1\right) V^{*} & =\left(\iota \otimes \Phi_{\rho(f)}^{r}\right) V(x \otimes 1) V^{*}=\left(\iota \otimes \Theta_{0}^{r}(f)\right) V(x \otimes 1) V^{*} \\
& =(\iota \otimes \iota \otimes f) V_{23} V_{12}(x \otimes 1 \otimes 1) V_{12}^{*} V_{23}^{*} \\
& =(\iota \otimes \iota \otimes f) V_{12} V_{13}(x \otimes 1 \otimes 1) V_{13}^{*} V_{12}^{*} \\
& =V\left(\Theta_{0}^{r}(f)(x) \otimes 1\right) V^{*} .
\end{aligned}
$$

Thus we get

$$
\Theta^{r}(\rho(f))(x)=(\iota \otimes f) V(x \otimes 1) V^{*}=\Theta_{0}^{r}(f)(x) .
$$

Finally, we would like to remark that we can, of course, analogously define a completely bounded left multiplier of $L_{1}(\mathbb{G})$ (associated with the left fundamental 
unitary operator $W)$ to be an element $\hat{b} \in \hat{M}$ such that $\hat{b} \lambda(f) \in \lambda\left(L_{1}(\mathbb{G})\right)$ for all $f \in L_{1}(\mathbb{G})$, and the induced map

$$
m_{\hat{b}}^{l}: f \in L_{1}(\mathbb{G}) \rightarrow \lambda^{-1}(\hat{b} \lambda(f))
$$

is completely bounded on $L_{1}(\mathbb{G})$. It is easy to show that $m_{\hat{b}}^{l}$ is actually a completely bounded left centralizer on $L_{1}(\mathbb{G})$, and we have

$$
m_{\hat{b}_{1} \hat{b}_{2}}^{l}=m_{\hat{b}_{1}}^{l} \circ m_{\hat{b}_{2}}^{l}
$$

for all $\hat{b}_{1}$ and $\hat{b}_{2}$ in $M_{\mathrm{cb}}^{l}\left(L_{1}(\mathbb{G})\right)$. If we let $M_{\mathrm{cb}}^{l}\left(L_{1}(\mathbb{G})\right)$ denote the algebra of all completely bounded left multipliers of $L_{1}(\mathbb{G})$, then we can obtain the following result.

Theorem 4.10. Let $\hat{b} \in \hat{M}$. Then $\hat{b}$ is contained in $M_{\mathrm{cb}}^{l}\left(L_{1}(\mathbb{G})\right)$ if and only if there exists a normal completely bounded map $\Phi_{\hat{b}}^{l}=\left(m_{\hat{b}}^{l}\right)^{*}$ on $M$ such that

$$
\left(\Phi_{\hat{b}}^{l} \otimes \iota\right)(W)=(1 \otimes \hat{b}) W .
$$

We have a completely isometric anti-isomorphism of completely contractive Banach algebras as follows:

$$
M_{\mathrm{cb}}^{l}\left(L_{1}(\mathbb{G})\right) \cong \mathcal{C B}_{\hat{M}^{\prime}}^{\sigma, M}\left(\mathcal{B}\left(L_{2}(\mathbb{G})\right)\right)
$$

\section{Pontryagin Duality as a Commutation Relation}

It is a well-known mathematical principle that many natural operations stabilize after two steps of iteration: negation in logic, dualizing of reflexive Banach spaces, forming the Pontryagin dual of locally compact abelian groups, and taking the commutant of a von Neumann algebra. The present section aims at showing that the latter pair of operations displays much more than a mere analogy. Indeed, we will prove that in the realm of locally compact quantum groups, Pontryagin duality is a commutation relation, in the framework of our representation theoretical model.

The following constitutes a generalization and unification of $[22$, Theorems 5.1 and 5.2]. Here, for a set $S \subseteq \mathcal{C B}\left(\mathcal{B}\left(L_{2}(\mathbb{G})\right)\right)$, we denote by $S^{c}$ its commutant in $\mathcal{C B}\left(\mathcal{B}\left(L_{2}(\mathbb{G})\right)\right)$.

Theorem 5.1. Let $\mathbb{G}$ be a locally compact quantum group and let $\hat{\mathbb{G}}$ denote the dual quantum group. Then we have

$$
\hat{\Theta}^{r}\left(M_{\mathrm{cb}}^{r}\left(L_{1}(\hat{\mathbb{G}})\right)\right)=\Theta^{r}\left(M_{\mathrm{cb}}^{r}\left(L_{1}(\mathbb{G})\right)\right)^{c} \cap \mathcal{C B}_{M}^{\sigma}\left(\mathcal{B}\left(L_{2}(\mathbb{G})\right)\right) .
$$

Proof. Due to Theorem 4.5, we have the identifications

$$
\Theta^{r}: M_{\mathrm{cb}}^{r}\left(L_{1}(\mathbb{G})\right) \simeq \mathcal{C B}_{\hat{M}}^{\sigma, M}\left(\mathcal{B}\left(L_{2}(\mathbb{G})\right)\right)
$$

and

$$
\hat{\Theta}^{r}: M_{\mathrm{cb}}^{r}\left(L_{1}(\hat{\mathbb{G}})\right) \simeq \mathcal{C B}_{M}^{\sigma, \hat{M}}\left(\mathcal{B}\left(L_{2}(\mathbb{G})\right)\right)
$$


"ᄃ" We show that

$$
\mathcal{C B}_{M}^{\sigma, \hat{M}}\left(\mathcal{B}\left(L_{2}(\mathbb{G})\right)\right) \subseteq \mathcal{C B}_{\hat{M}}^{\sigma, M}\left(\mathcal{B}\left(L_{2}(\mathbb{G})\right)\right)^{c} \cap \mathcal{C B}_{M}^{\sigma}\left(\mathcal{B}\left(L_{2}(\mathbb{G})\right)\right) .
$$

Let $\Phi \in \mathcal{C B}_{M}^{\sigma, \hat{M}}\left(\mathcal{B}\left(L_{2}(\mathbb{G})\right)\right)$ and $\Psi \in \mathcal{C B}_{\hat{M}}^{\sigma, M}\left(\mathcal{B}\left(L_{2}(\mathbb{G})\right)\right)$. Then, since both maps are normal and the linear span of $\hat{M} M$ is weak*-dense in $\mathcal{B}\left(L_{2}(\mathbb{G})\right)$ by our Proposition 2.2, we only need to show that $\Phi \Psi(\hat{x} y)=\Psi \Phi(\hat{x} y)$ for all $\hat{x} \in \hat{M}$ and $y \in M$. On the one hand, since $\Psi$ is an $\hat{M}$-bimodule map and leaves $M$ invariant, and finally using the $M$-bimodule property of $\Phi$, we obtain

$$
\Phi \Psi(\hat{x} y)=\Phi(\hat{x} \Psi(y))=\Phi(\hat{x}) \Psi(y) .
$$

On the other hand, since $\Phi$ is an $M$-bimodule map and leaves $\hat{M}$ invariant, and using the $\hat{M}$-bimodule property of $\Psi$, we get analogously:

$$
\Psi \Phi(\hat{x} y)=\Psi(\Phi(\hat{x}) y)=\Phi(\hat{x}) \Psi(y)
$$

which yields the desired equality.

"" The argument is similar to the one given in the proof of $[22$, Theorem 5.1].

Let $\Phi \in \mathcal{C B}_{\hat{M}}^{\sigma, M}\left(\mathcal{B}\left(L_{2}(\mathbb{G})\right)\right)^{c} \cap \mathcal{C B}_{M}^{\sigma}\left(\mathcal{B}\left(L_{2}(\mathbb{G})\right)\right)$. It sufficies to show that $\Phi$ leaves $\hat{M}$ invariant. Since $\rho\left(L_{1}(\mathbb{G})\right)$ is weak ${ }^{*}$ dense in $\hat{M}^{\prime}$, it sufficies to show that

$$
\rho(f) \Phi(\hat{x})=\Phi(\hat{x}) \rho(f)
$$

for all $f \in L_{1}(\mathbb{G})=M_{*}$ and $\hat{x} \in \hat{M}$. Assume that $\left\{e_{i}\right\}$ is an orthonormal basis for $L_{2}(\mathbb{G})$. Then we let $\omega_{i j}=\theta_{e_{i}}^{*} \theta_{e_{j}} \in \mathcal{T}\left(L_{2}(\mathbb{G})\right)$ and $f_{i j}=\left.\omega_{i j}\right|_{M} \in L_{1}(\mathbb{G})$. Since $\Theta^{r}\left(f_{i j}\right)$ is an $\hat{M}$-bimodule map, we have

$$
\Phi\left(\Theta^{r}\left(f_{i j}\right)(\hat{x})\right)=\left\langle f_{i j}, 1\right\rangle \Phi(\hat{x}) .
$$

(Note that this is precisely an abstract version of [22, Eq. (5.2)].) Therefore, we obtain

$$
\begin{aligned}
\left\langle\Phi(\hat{x}) \otimes 1, \iota \otimes f_{i j}\right\rangle & =\left\langle f_{i j}, 1\right\rangle \Phi(\hat{x})=\Phi\left(\Theta^{r}\left(f_{i j}\right)(\hat{x})\right)=\Theta^{r}\left(f_{i j}\right)(\Phi(\hat{x})) \\
& =\left\langle V(\Phi(\hat{x}) \otimes 1) V^{*}, \iota \otimes f_{i j}\right\rangle .
\end{aligned}
$$

This shows that

$$
\Phi(\hat{x}) \otimes 1=V(\Phi(\hat{x}) \otimes 1) V^{*}
$$

or equivalently,

$$
(\Phi(\hat{x}) \otimes 1) V=V(\Phi(\hat{x}) \otimes 1) .
$$

For any $f \in L_{1}(\mathbb{G})$, we apply $\iota \otimes f$ to the above identity and get

$$
\Phi(\hat{x}) \rho(f)=\rho(f) \Phi(\hat{x}) .
$$


This shows that $\Phi(\hat{x}) \in \hat{M}$ and thus $\Phi$ is a normal completely map contained in $\mathcal{C B}_{M}^{\sigma, \hat{M}}\left(\mathcal{B}\left(L_{2}(\mathbb{G})\right)\right)=\hat{\Theta}^{r}\left(M_{\mathrm{cb}}^{r}\left(L_{1}(\hat{\mathbb{G}})\right)\right)$. This completes the proof.

Remark 5.2. (i) The following question is crucial for the interpretation of Theorem 5.1 beyond the realm of compact quantum groups $\mathbb{G}$ (for which $M_{\mathrm{cb}}^{r}\left(L_{1}(\hat{\mathbb{G}})\right)=$ $\left.\rho\left(L_{1}(\hat{\mathbb{G}})\right)\right)$ : For quantum groups, does $M_{\mathrm{cb}}^{r}\left(L_{1}(\mathbb{G})\right)$ determine $\mathbb{G}$, i.e. if $\mathbb{G}_{1}$ and $\mathbb{G}_{2}$ are locally compact quantum groups such that $M_{\mathrm{cb}}^{r}\left(L_{1}\left(\mathbb{G}_{1}\right)\right)$ and $M_{\mathrm{cb}}^{r}\left(L_{1}\left(\mathbb{G}_{2}\right)\right)$ are completely isometrically algebra isomorphic, is the same true for $L_{1}\left(\mathbb{G}_{1}\right)$ and $L_{1}\left(\mathbb{G}_{2}\right)$ ? If $G$ is a locally compact group, this is true for the measure algebra $M(G)=M_{\mathrm{cb}}^{r}\left(L_{1}\left(\mathbb{G}_{a}\right)\right)$, and when $G$ is amenable, this is also true for the completely bounded (Herg-Schur) multiplier algebra $M_{\mathrm{cb}} A(G)=M_{\mathrm{cb}}^{r}\left(L_{1}\left(\hat{\mathbb{G}}_{a}\right)\right)$ since, in this case, $M_{\mathrm{cb}} A(G)$ is equal to the Fourier-Stieltjes algebra $B(G)$. So it would be interesting to know whether this question has a positive answer for co-amenable locally compact quantum groups.

(ii) Theorem 5.1, together with the above remark (i), also prompts the question whether the quantum group version $\hat{\hat{G}}=\mathbb{G}$ of the classical Pontryagin duality theorem (via the dual quantum group $\hat{\mathbb{G}}$ ) may translate, on the representation side, into the bicommutant result $\Theta^{r}\left(M_{\mathrm{cb}}^{r}\left(L_{1}(\mathbb{G})\right)\right)^{c c}=\Theta^{r}\left(M_{\mathrm{cb}}^{r}\left(L_{1}(\mathbb{G})\right)\right)$, where commutants are taken in $\mathcal{C B}\left(\mathcal{B}\left(L_{2}(\mathbb{G})\right)\right)$.

Corollary 5.3. Let $\mathbb{G}$ be a locally compact quantum group with dual $\hat{\mathbb{G}}$. Then we have the bicommutant result

$$
\Theta^{r}\left(M_{\mathrm{cb}}^{r}\left(L_{1}(\mathbb{G})\right)\right)^{c c}=\Theta^{r}\left(M_{\mathrm{cb}}^{r}\left(L_{1}(\mathbb{G})\right)\right),
$$

or equivalently,

$$
\mathcal{C B}_{\hat{M}}^{\sigma, M}\left(\mathcal{B}\left(L_{2}(\mathbb{G})\right)\right)^{c c}=\mathcal{C B}_{\hat{M}}^{\sigma, M}\left(\mathcal{B}\left(L_{2}(\mathbb{G})\right)\right)
$$

Proof. This follows in the same way as [22, Theorem 5.4], by using the identification

$$
\Theta^{r}\left(M_{\mathrm{cb}}^{r}\left(L_{1}(\mathbb{G})\right)\right)=\mathcal{C B}_{\hat{M}}^{\sigma, M}\left(\mathcal{B}\left(L_{2}(\mathbb{G})\right)\right)
$$

and the Effros-Exel/Hofmeier-Wittstock bicommutant theorem ([6, Sec. 3], [11, Proposition 3.1 and Remark 4.3])

$$
\mathcal{C B}_{N}^{\sigma}(\mathcal{B}(H))^{c c}=\mathcal{C B}_{N}^{\sigma}(\mathcal{B}(H))
$$

which holds for every von Neumann subalgebra $N \subseteq \mathcal{B}(H)$ in standard form, where $H$ is a (separable) Hilbert space; the extension to arbitrary Hilbert spaces follows from [17, Sec. 2].

\section{Acknowledgments}

The first and third authors were partially supported by the National Science Foundation (NSF) DMS 05-56120 and DMS 05-00535. The second author was partially 
supported by the Natural Sciences and Engineering Research Council of Canada (NSERC) 261894-2003.

Finally, the first and the third named authors wish to express their particular thanks to L. Vainerman and A. Van Daele for helpful discussions on locally compact quantum groups.

\section{References}

[1] S. Baaj and G. Skandalis, Unitaires multiplicatifs et dualité pour les produits croisés de $C^{*}$-algèbres, Ann. Sci. École Norm. Sup. 26 (1993) 425-488.

[2] S. Baaj, G. Skandalis and S. Vaes, Non-semi-regular quantum groups coming from number theory, Commun. Math. Phys. 235 (2003) 139-167.

[3] D. P. Blecher and D. M. Hay, Complete isometries - an illustration of noncommutative functional analysis, in Function Spaces (Edwardsville, IL, 2002), Contemporary Mathematics, Vol. 328 (American Mathematical Society, Providence, RI, 2003), pp. 85-97.

[4] J. De Cannière, On the intrinsic group of a Kac algebra, Proc. London Math. Soc. (3) 40(1) (1980) 1-20.

[5] V. G. Drinfel'd, Quantum groups, in Proceedings of the ICM Berkeley (1986), pp. $57-100$.

[6] E. Effros and R. Exel, On Multilinear Double Commutant Theorems, Operator algebras and applications, Vol. 1, 81-94, London Math. Soc. Lecture Note Ser. 135 (Cambridge Univ. Press, Cambridge, 1988).

[7] E. Effros and Z.-J. Ruan, Discrete quantum groups. I. The Haar measure, Int. J. Math. 5 (1994) 681-723.

[8] E. G. Effros and Z.-J. Ruan, Operator Spaces, London Mathematical Society Monographs, New Series, Vol. 23 (Oxford University Press, New York, 2000).

[9] M. Enock and J.-M. Schwartz, Kac Algebras and Duality of Locally Compact Groups (Springer-Verlag, Berlin-Heidelberg, 1992).

[10] U. Haagerup, Decomposition of completely bounded maps on operator algebras, unpublished manuscript, 1980.

[11] H. Hofmeier and G. Wittstock, A bicommutant theorem for completely bounded module homomorphisms, Math. Ann. 308 (1997) 141-154.

[12] B. E. Johnson, An introduction to double centralizers, Proc. London Math. Soc. (3) 14 (1964) 299-320.

[13] M. Kalantar and M. Neufang, The intrinsic group of a locally compact quantum group, preprint.

[14] J. Kraus and Z.-J. Ruan, Multipliers of Kac algebras, Int. J. Math. 8 (1997) 213-248.

[15] J. Kustermans and S. Vaes, Locally compact quantum groups, Ann. Sci. Ecole Norm. Sup. 33 (2000) 837-934.

[16] J. Kustermans and S. Vaes, Locally compact quantum groups in the von Neumann algebraic setting, Math. Scand. 92 (2003) 68-92.

[17] B. Magajna, On completely bounded bimodule maps over $W^{*}$-algebras, Studia Math. 154 (2003) 137-164.

[18] M. Neufang, Abstrakte harmonische Analyse und Modulhomomorphismen über von Neumann-Algebren, Ph.D. thesis, Universität des Saarlandes (2000).

[19] M. Neufang, Isometric representations of convolution algebras as completely bounded module homomorphisms and a characterization of the measure algebra, preprint.

[20] M. Neufang, Bicommutant theorems in the operator space $\mathcal{C B}(\mathcal{B}(\mathcal{H}))$, II - Automatic normality and a non-commutative version of the Brainerd-Edwards Theorem, preprint. 
[21] Z. Hu, M. Neufang and Z.-J. Ruan, Completely bounded multipliers on co-amenable locally compact quantum groups, in preparation.

[22] M. Neufang, Z.-J. Ruan and N. Spronk, Completely isometric representations of $M_{\mathrm{cb}} A(G)$ and $U C B(\hat{G})^{*}$, Trans. Amer. Math. Soc. 360 (2008) 1133-1161.

[23] G. Pisier, Non-commutative vector valued $L_{p}$-spaces and completely $p$-summing maps, Astérisque 247 (1998).

[24] G. Pisier, An Introduction to the Theory of Operator Spaces, London Mathematical Society Lecture Note Series, Vol. 294 (Cambridge University Press, Cambridge, 2003).

[25] A.-M. Popa and J.-Z. Ruan, Uniformly complete quotient space $U C Q(G)$ and completely isometric representations of $U C Q(G)^{*}$ on $B\left(L_{2}(G)\right)$, Proc. Amer. Math. Soc. 134 (2005) 1223-1235.

[26] S. Sakai, $C^{*}$-Algebras and $W^{*}$-Algebras (Springer, 1998).

[27] S. Vaes, Locally compact quantum groups, Ph.D. thesis, Katholieke Universitiet Leuven (2001).

[28] S. Vaes and A. Van Daele, The Heisenberg commutation relations, commuting squares and the Haar measure on locally compact quantum groups, in Operator Algebras and Mathematical Physics (Constanta, 2001) (Theta, Bucharest, 2003), pp. 379-400.

[29] A. Van Daele, Discrete quantum groups, J. Algebra 180 (1996) 431-444.

[30] A. Van Daele, Locally compact quantum groups. A von Neumann algebra approach, preprint.

[31] J. Wendel, Left centralizers and isomorphisms of group algebras, Pacific J. Math. 2 (1952) 251-261.

[32] S. L. Woronowicz, Twisted $S U(2)$ group. An example of a non-commutative diffferential calculus, Publ. Res. Inst. Math. Sci. 23 (1987) 117-181.

[33] S. L. Woronowicz, From multiplicative unitaries to quantum groups, Int. J. Math. 7 (1996) 127-149.

[34] S. L. Woronowicz, Compact quantum groups, in Symétries Quantiques (Les Houches, 1995), (North-Holland, Amsterdam, 1998), pp. 845-884. 